\title{
Comparative Examination of the Olive Mill Wastewater Biodegradation Process by Various Wood-Rot Macrofungi
}

\author{
Georgios Koutrotsios and Georgios I. Zervakis \\ Laboratory of General and Agricultural Microbiology, Agricultural University of Athens, Iera Odos 75, 11855 Athens, Greece \\ Correspondence should be addressed to Georgios I. Zervakis; zervakis@aua.gr
}

Received 27 February 2014; Revised 30 April 2014; Accepted 1 May 2014; Published 2 June 2014

Academic Editor: Dawen Gao

Copyright ( 2014 G. Koutrotsios and G. I. Zervakis. This is an open access article distributed under the Creative Commons Attribution License, which permits unrestricted use, distribution, and reproduction in any medium, provided the original work is properly cited.

\begin{abstract}
Olive mill wastewater (OMW) constitutes a major cause of environmental pollution in olive-oil producing regions. Sixty wood-rot macrofungi assigned in 43 species were evaluated for their efficacy to colonize solidified OMW media at initially established optimal growth temperatures. Subsequently eight strains of the following species were qualified: Abortiporus biennis, Ganoderma carnosum, Hapalopilus croceus, Hericium erinaceus, Irpex lacteus, Phanerochaete chrysosporium, Pleurotus djamor, and P. pulmonarius. Fungal growth in OMW $(25 \% \mathrm{v} / \mathrm{v}$ in water) resulted in marked reduction of total phenolic content, which was significantly correlated with the effluent's decolorization. A. biennis was the best performing strain (it decreased phenolics by $92 \%$ and color by $64 \%$ ) followed by $P$. djamor and I. lacteus. Increase of plant seeds germination was less pronounced evidencing that phenolics are only partly responsible for OMW's phytotoxicity. Laccase production was highly correlated with all three biodegradation parameters for $H$. croceus, Ph. chrysosporium, and Pleurotus spp., and so were manganese-independent and manganese dependent peroxidases for $A$. biennis and I. lacteus. Monitoring of enzymes with respect to biomass production indicated that Pleurotus spp., $\mathrm{H}$. croceus, and $\mathrm{Ph}$. chrysosporium shared common patterns for all three activities. Moreover, generation of enzymes at the early biodegradation stages enhanced the efficiency of OMW treatment.
\end{abstract}

\section{Introduction}

Three-phase centrifugal olive mills are widespread in most olive-oil producing countries and their operation results in the production of olive oil, solid pomace, and olive mill wastewater (OMW). The disposal of the latter creates major environmental problems since it is rich in organic compounds and contains large amounts of polyphenolics in high (and therefore) toxic concentrations [1,2]. In addition, the olive mills seasonal operation, small size, and scattered distribution hinder the effective management of the effluent [3].

Among the large variety of methodologies adopted for the treatment of OMW [4-7], implementation of biological approaches is of particular importance. For example, the use of microorganisms could result in both the detoxification of the effluent and the generation of value-added products, for example, xanthan gum, citric acid, ethanol, antioxidants, polyhydroxyalkanoates, b-glucan, lipase, single cell oil, and edible biomass [8-18].
In particular, wood-rot macrofungi constitute a group of organisms equipped with a potent biochemical arsenal including one or more nonspecific groups of enzymes, which permits them to effectively decompose various lignocellulosic compounds [19-21]. The chemical affinity of the latter to a wide range of agroindustrial wastes (including OMW) led to their treatment with filamentous basidiomycetes originally isolated from woody substrates. Therefore, such organisms were examined either at a OMW pretreatment stage for decreasing the effluent's high content of phenolics and hence toxicity prior to its processing with other microorganisms $[22,23]$ or as the main/sole agents for OMW's remediation [24-27]. However, most pertinent investigations have focused on the exploitation of a few species only, for example, Phanerochaete chrysosporium, Ph. flavido-alba, Pleurotus ostreatus, and P. eryngii [28-32], and consequently a large number of other potentially useful macrofungi remain understudied or not examined at all. 
The objective of the present work was the comparative evaluation of a wide variety of mushroom fungi for OMW biotreatment and the subsequent establishment of a battery of strains able to efficiently degrade OMW. The process was assessed by determining the activities of ligninolytic enzymes produced by qualified organisms at different time periods and through their association with concomitant OMW decolorization, reduction of phenolics, and decrease of plant toxicity.

\section{Materials and Methods}

2.1. Fungal Strains. Sixty wood-rot macrofungi belonging to 43 species of basidiomycetes (phylum Basidiomycota) were evaluated for the purposes of this study. Details of the strains identity are presented in Table 1 . All the biological material is maintained in the fungal culture collection of the authors institution (AUA-LGAM).

\subsection{Nutrient Media: Fungal Growth Substrates and Condi-} tions. OMW was obtained from an olive-oil mill equipped with three-phase centrifugal decanters located in Kalamata (Peloponnese, S.W. Greece). Its composition and main physicochemical properties were previously assessed [2].

For the preparation of OMW-containing growth media, the effluent was adjusted at $\mathrm{pH} 6$ by adding $\mathrm{CaO}$ and centrifuged for $20 \mathrm{~min}$ at $5000 \mathrm{~g}\left(5^{\circ} \mathrm{C}\right)$, and the supernatant was diluted or not with deionized water to prepare $25 \%, 50 \%$, and $100 \% \mathrm{v} / \mathrm{v}$ OMW-based substrates, which were further heat-sterilized for $30 \mathrm{~min}\left(121^{\circ} \mathrm{C}, 1.1 \mathrm{~atm}\right)$. Fungal strains were subcultured on a $25 \% \mathrm{v} / \mathrm{v}$ OMW medium solidified with $1.7 \%$ w/v agar. Then, agar plugs (6 mm diam.) originating from the actively growing part of the fungal colonies were used to inoculate either solidified OMW media (for the purposes of mycelium growth rate determinations) or static liquid cultures (for OMW degradation experiments with selected strains).

\subsection{Screening of Fungal Strains: Establishment of Temperature} Optima and Measurements of Linear Growth Rates. The temperature optima for mycelium linear growth were assessed on potato dextrose agar (PDA; Conda) medium and over the range of $12-47^{\circ} \mathrm{C}$ with a $5^{\circ} \mathrm{C}$ graduation. Experiments were conducted in Petri dishes as previously described [33], and measurements were taken every $24 \mathrm{~h}$ for all species examined with the exception of Phanerochaete chrysosporium and Hapalopilus croceus strains whose growth was measured every $12 \mathrm{~h}$ until seven consecutive values were obtained for all strains.

Growth rates were determined by measuring the distance of the colony's front from the centre of the inoculum at four different points along two perpendicular lines [34]. Mycelium growth rates $\left(k_{r}\right)$ were calculated by fitting the linear growth function $y=k_{r} x+c$ (where $y$ is the distance covered by the hyphae and $x$ is the respective time) and were expressed in $\mathrm{mm} \mathrm{d}^{-1}$. All experiments were conducted in three replicates.

After establishing the optimal temperature for mycelium growth for each strain, all cultures were evaluated with respect to their growth rates on various OMW dilutions (25\%, $50 \%$, and $100 \% \mathrm{v} / \mathrm{v}$ OMW in water) at their optimal temperatures as previously determined, in the same way as described above. In addition, PDA was used as control. All mycelium growth rate experiments were conducted in three replicates.

2.4. Treatment of OMW by Qualified Fungal Strains: Assessment of Effluent's Selected Properties. The strains, which were qualified from the previous experiment, were further examined as regards their effect on OMW degradation process in liquid static cultures. The latter were performed in $250 \mathrm{~mL}$ Erlenmayer flasks containing $100 \mathrm{~mL}$ of $25 \% \mathrm{v} / \mathrm{v}$ OMW, at the optimal temperatures previously established for each strain, and lasted for a period from 20 to 30 days depending on the growth of each fungus, that is, Abortiporus biennis ABL436 (25 days), Ganoderma carnosum GCL448 (25 days), Hapalopilus croceus HCC522 (20 days), Hericium erinaceus HEL801 (25 days), Irpex lacteus ILC238 (25 days), Phanerochaete chrysosporium PHL322 (20 days), Pleurotus djamor PDC855 (30 days), and P. pulmonarius PPL111 (30 days). Four replicates were included for each treatment, while noninoculated substrates were also incubated in parallel and served as controls.

A destructive sampling process was adopted at five time points for dividing the entire cultivation at intervals of four, five, or six days (for growth periods of 20, 25, or 30 days, resp.), in order to determine $\mathrm{pH}$, electric conductivity, biomass, total phenolics, decolorization, plant seed germination, and enzymes activity.

Measurements of $\mathrm{pH}$ and electric conductivity in OMW were performed by using a Corning EEL $12 \mathrm{pH}$ meter and a Jenway 4010 conductivity meter, respectively. Biomass was harvested by filtration and its weight (mycelium dry weight) was determined by drying at $60^{\circ} \mathrm{C}$ until constant weight.

Total phenolics were analyzed by the Folin-Ciocalteu method [35], by measuring spectrophotometrically (at $760 \mathrm{~nm}$ ) the formation of a blue complex as a result of the reduction of a phosphomolybdic-phosphotungstic reagent from the presence of phenolics. The concentration of total phenolics was determined against a syringic acid calibration curve $\left(1 \mathrm{mg} \mathrm{mL}^{-1}\right.$ syringic acid-initial concentrationresulted in an optical density of 0.377 at $760 \mathrm{~nm}$ ).

Decolorization was estimated by measuring the absorbance of OMW samples at $525 \mathrm{~nm}$ using a U-2001 spectrophotometer (Hitachi Instruments Inc., USA).

The plant seed germination indexes were estimated on the basis of Zucconi et al. [36] protocol. Twenty-five cress seeds (Lepidium sativum L.) were placed on filter papers moistened with OMW (25\%) and incubated in Petri dishes for three days at $25^{\circ} \mathrm{C}$. The effect of OMW on germination was evaluated, and germination indexes (G.I.) were calculated as follows: G.I. $=(\%$ root elongation $\times \%$ germination $) / 100$ (control used: cress seeds moistened with water).

2.5. Determination of Enzymes Activities. Laccase (Lac, E.C. 1.10.3.2: benzenediol: oxygen oxidoreductase) activity was determined at $425 \mathrm{~nm}$ by oxidizing $0.4 \mathrm{~mL}$ ABTS (2,2-azinobis(3-ethylbenzothiazoline-6-sulphonic acid)) (1.5 mM) with 
TABLE 1: Details of the biological material evaluated for the purposes of this study.

\begin{tabular}{|c|c|c|}
\hline Species & Geographic origin and host/substrate & Collection code \\
\hline Abortiporus biennis & Czech Republic & ABC521 \\
\hline Abortiporus biennis & Greece & ABL436 \\
\hline Agrocybe cylindracea & China & ACL834 \\
\hline Auricularia mesenterica & Greece; Cupressus sp. & AML472 \\
\hline Coriolopsis trogii & Greece; Quercus sp. & CTL447 \\
\hline Daedalea quercina & Czech Republic & DQC528 \\
\hline Dichomitus squalens & Czech Republic & DSC750 \\
\hline Fistulina hepatica & Greece; Castanea sativa & FHL295 \\
\hline Flammulina velutipes & Commercial strain & FVS803 \\
\hline Fomitopsis pinicola & Greece; Abies cephalonica & FPL302 \\
\hline Ganoderma adspersum & Greece; Abies cephalonica & GAL401 \\
\hline Ganoderma carnosum & Greece & GCL642 \\
\hline Ganoderma carnosum & Greece; Olea sativa & GCL448 \\
\hline Ganoderma pfeifferi & Greece; Fagus sylvatica & GPL336 \\
\hline Ganoderma resinaceum & Greece; Morus alba & GRL334 \\
\hline Ganoderma resinaceum & Greece; Salix babylonica & GRL403 \\
\hline Ganoderma resinaceum & Czech Republic & GRC604 \\
\hline Grifola frondosa & Commercial strain & GFS805 \\
\hline Hapalopilus croceus & Czech Republic & HCC522 \\
\hline Hericium erinaceus & Greece & HEL801 \\
\hline Hericium erinaceus & Greece; Quercus pubescens & HEL802 \\
\hline Heterobasidion annosum & Greece; Abies cephalonica & HAL340 \\
\hline Hypsizygus ulmarius & Greece; Abies cephalonica & HUL417 \\
\hline Inocutis tamaricis & Greece; Tamarix hampeana & ITL314 \\
\hline Irpex lacteus & Czech Republic & ILC238 \\
\hline Laetiporus sulphureus & Greece; Castanea sativa & LSL331 \\
\hline Laetiporus sulphureus & Greece; Castanea sativa & LSL332 \\
\hline Lentinula edodes & Commercial strain & LES812 \\
\hline Neolentinus lepideus & Greece; Pinus nigra & NLL317 \\
\hline Omphalotus illudens & Greece; Quercus sp. & OIL347 \\
\hline Perenniporia fraxinea & Greece; Populus alba & PFL346 \\
\hline Phanerochaete chrysosporium & Greece & PHL322 \\
\hline Phanerochaete chrysosporium & Czech Republic & PHC571 \\
\hline Pholiota nameko & commercial strain & PNS806 \\
\hline Pleurotus abieticola & Russia & PAC854 \\
\hline Pleurotus citrinopileatus & Malaysia & PCC884 \\
\hline Pleurotus cornucopiae & Iran & PCL660 \\
\hline Pleurotus cystidiosus & USA; Populus deltoides & PCC897 \\
\hline Pleurotus cystidiosus subsp. abalonus & China & PAC891 \\
\hline Pleurotus djamor & Malaysia & PDC855 \\
\hline Pleurotus eryngii & Czech Republic & PEC847 \\
\hline Pleurotus eryngii var. eryngii & Greece; Eryngium sp. & PEL063 \\
\hline Pleurotus eryngii var. eryngii & Italy; Eryngium campestre & PEC810 \\
\hline Pleurotus eryngii subsp. tuoliensis & China & PEC856 \\
\hline Pleurotus flabellatus & Malaysia & PFC860 \\
\hline Pleurotus nebrodensis & Greece; Prangos ferulacea & PNL126 \\
\hline Pleurotus nebrodensis & Italy; Prangos ferulacea & PNC816 \\
\hline Pleurotus ostreatus & Italy & POC847 \\
\hline Pleurotus ostreatus & Czech Republic & POC843 \\
\hline
\end{tabular}


TABle 1: Continued.

\begin{tabular}{lcc}
\hline Species & Geographic origin and host/substrate & Collection code \\
\hline Pleurotus ostreatus & Greece; Abies cephalonica & POL067 \\
Pleurotus pulmonarius & Greece; Fagus sylvatica & PPL111 \\
Pleurotus pulmonarius & France & PPC823 \\
Pleurotus pulmonarius & Hong Kong & PSC757 \\
Pleurotus tuber-regium & Papua New Guinea & PTC822 \\
Stereum hirsutum & Czech Republic & SHC608 \\
Trametes hirsuta & Czech Republic & THC610 \\
Trametes ljubarskyi & Greece; Populus sp. & TLL473 \\
Trametes versicolor & Greece & TVL469 \\
Trametes versicolor & Czech Republic & TVC614 \\
Tyromyces lacteus & Czech Republic & TLC616 \\
\hline
\end{tabular}

$0.8 \mathrm{~mL}$ of OMW sample in $1.2 \mathrm{~mL}$ Na-tartrate buffer $(0.1 \mathrm{M}$, $\mathrm{pH} 4.5)$ [37].

Manganese-independent peroxidase (MnIP) was measured at $590 \mathrm{~nm}$ by the oxidative coupling of $0.1 \mathrm{~mL} \mathrm{MBTH}$ (3-methyl-2-benzothiazoline hydrazone) $(1 \mathrm{mM})$ and $0.2 \mathrm{~mL}$ DMAB (3-dimethylaminobenzoic acid) $(25 \mathrm{mM})$ in the presence of $0.01 \mathrm{~mL} \mathrm{H}_{2} \mathrm{O}_{2}(10 \mathrm{mM})$ added to a solution consisting of $0.66 \mathrm{~mL}$ sample and $1 \mathrm{~mL}$ succinate-lactate buffer $(0.1 \mathrm{M}$, $\mathrm{pH} 4.5$ ), while background activity, determined as above in the absence of $\mathrm{H}_{2} \mathrm{O}_{2}$, was subtracted [32].

Manganese peroxidase (MnP, E.C. 1.11.1.13 (MnII): hydrogen peroxide oxidoreductase) activity was determined as described for manganese-independent peroxidase (MnIP) in the presence of $0.01 \mathrm{~mL} \mathrm{MnSO}_{4}(20 \mathrm{mM})$, by subtracting MnIP activity [38]. For all enzymes determination, one activity unit was defined as the amount of enzyme transforming $1 \mu \mathrm{mol}$ of substrate per minute.

2.6. Statistical Analysis. Analysis of variance (ANOVA) followed by Gabriel's multiple comparison tests $(a<0.05)$ was used to estimate statistical differences between treatment means through the use of SPSS (version 18) software. Standard deviations were calculated for all mean values, and regression analysis was carried out to evaluate relationships between variables at significance levels of $1 \%$ and $5 \%$.

\section{Results and Discussion}

Data about the optimal conditions for the production of fungal biomass are available for a rather few species of macrofungi (i.e., mainly those exploited for edible mushroom production). The effect of temperature, in particular, is of fundamental importance in evaluating the biotechnological potential of such organisms $[33,34]$, and hence it was the first parameter which was assessed in the present study. The results of the comparative mycelium linear growth rate examination covering a wide temperature range (12$47^{\circ} \mathrm{C}$ ) showed that most strains presented their optima at a narrow value area of $27-32^{\circ} \mathrm{C}$ (Table 2). Only Hypsizygus ulmarius, Omphalotus illudens, Pholiota nameko, and Pleurotus nebrodensis exhibited significantly higher growth rates at $22^{\circ} \mathrm{C}$. On the other hand, Phanerochaete chrysosporium

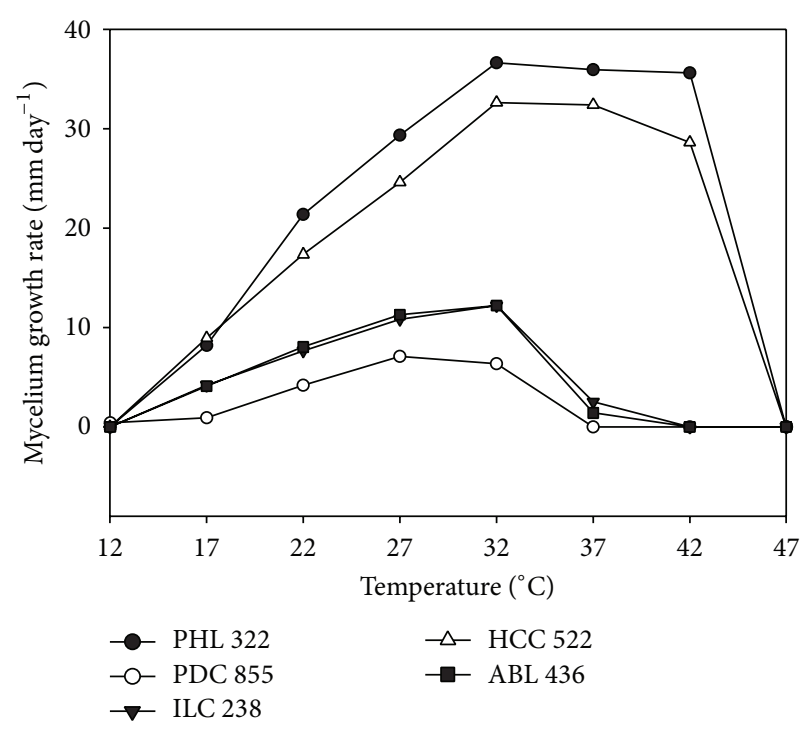

FIgURE 1: Mycelium linear growth rates $\left(K_{r}, \mathrm{~mm} \mathrm{day}^{-1}\right)$ on PDA as measured at different temperatures $\left({ }^{\circ} \mathrm{C}\right)$ for selected indicative cases of macrofungi examined (i.e., A. biennis ABL436, $H$. croceus HCC522, I. lacteus ILC238, Ph. chrysosporium PHL322, and P. djamor PDC855).

PHC571 was the only one presenting optimal growth at $37^{\circ} \mathrm{C}$. It is noteworthy that almost all Pleurotus strains showed a growth (albeit limited) at $12^{\circ} \mathrm{C}$; Pleurotus was the only genus among the 28 different genera evaluated demonstrating such a property. In contrast, Hapalopilus croceus and Phanerochaete chrysosporium were the only species showing hyphal development at the other temperature extreme $\left(42^{\circ} \mathrm{C}\right)$. Furthermore, mycelium growth rates of almost all strains presented a gradual increase from lower temperatures to the optimal one and then a sharp decrease to the temperature of no growth; in fact, this effect was more pronounced for individuals demonstrating high growth values (Figure 1).

In the next comparative evaluation test, the mycelium growth rates of all strains were measured at their optimal temperatures and on three different OMW concentrations (25\%, $50 \%$, and $100 \%$ OMW v/v, in water) plus on PDA (Table 3 ). 
TABLE 2: Mycelium linear growth rates $\left(K_{r}, \mathrm{~mm} \mathrm{day}^{-1}\right)$ for 60 strains of macrofungi established at different temperatures ranging from 12 to $47^{\circ} \mathrm{C}$ (ngd: no growth detected). Values are expressed as means \pm standard deviation of means, $n=3$. Lack of superscript letters commonly indicates statistically significant differences (Gabriel's $t$-test, $P<0.05$ ) for comparisons of treatment means between different temperatures for each strain.

\begin{tabular}{|c|c|c|c|c|c|c|c|c|}
\hline \multirow{2}{*}{ Strain } & \multicolumn{8}{|c|}{ Temperature } \\
\hline & 12 & 17 & 22 & 27 & 32 & 37 & 42 & 47 \\
\hline ABC521 & ngd & $4.06 \pm 0.04^{\mathrm{d}}$ & $7.46 \pm 0.07^{\mathrm{c}}$ & $11.47 \pm 0.07^{\mathrm{a}}$ & $10.17 \pm 0.07^{\mathrm{b}}$ & $1.47 \pm 0.07^{\mathrm{e}}$ & ngd & ngd \\
\hline ABL436 & ngd & $4.10 \pm 0.18^{\mathrm{d}}$ & $8.03 \pm 0.03^{c}$ & $11.30 \pm 0.08^{\mathrm{b}}$ & $12.19 \pm 0.29^{\mathrm{a}}$ & $1.39 \pm 0.08^{\mathrm{e}}$ & ngd & ngd \\
\hline ACL834 & ngd & $1.64 \pm 0.04^{c}$ & $3.12 \pm 0.13^{\mathrm{b}}$ & $4.02 \pm 0.10^{\mathrm{a}}$ & $3.06 \pm 0.08^{\mathrm{b}}$ & ngd & ngd & ngd \\
\hline AML472 & ngd & ngd & $6.24 \pm 0.17^{\mathrm{b}}$ & $6.97 \pm 0.30^{\mathrm{a}}$ & $5.62 \pm 0.29^{c}$ & ngd & ngd & ngd \\
\hline CTL447 & ngd & $2.60 \pm 0.08^{\mathrm{e}}$ & $4.64 \pm 0.15^{\mathrm{d}}$ & $6.51 \pm 0.13^{c}$ & $8.98 \pm 0.15^{\mathrm{a}}$ & $7.29 \pm 0.02^{\mathrm{b}}$ & ngd & ngd \\
\hline DQC528 & ngd & $3.94 \pm 0.14^{\mathrm{e}}$ & $7.03 \pm 0.22^{c}$ & $7.81 \pm 0.04^{\mathrm{b}}$ & $11.14 \pm 0.17^{\mathrm{a}}$ & $4.34 \pm 0.05^{\mathrm{d}}$ & ngd & ngd \\
\hline DSC750 & ngd & $3.52 \pm 0.13^{c}$ & $7.07 \pm 0.04^{\mathrm{b}}$ & $10.96 \pm 0.17^{\mathrm{a}}$ & $11.10 \pm 0.12^{\mathrm{a}}$ & $3.77 \pm 0.04^{c}$ & ngd & ngd \\
\hline FHL295 & ngd & $0.78 \pm 0.02^{\mathrm{b}}$ & $2.10 \pm 0.04^{\mathrm{a}}$ & $1.91 \pm 0.23^{\mathrm{a}}$ & $0.66 \pm 0.04^{\mathrm{b}}$ & ngd & ngd & ngd \\
\hline FVS803 & ngd & $3.66 \pm 0.15^{c}$ & $5.39 \pm 0.03^{\mathrm{b}}$ & $6.00 \pm 0.14^{\mathrm{a}}$ & $1.35 \pm 0.11^{\mathrm{d}}$ & ngd & ngd & ngd \\
\hline FPL302 & ngd & $2.34 \pm 0.02^{\mathrm{d}}$ & $5.55 \pm 0.02^{c}$ & $7.61 \pm 0.18^{\mathrm{b}}$ & $7.94 \pm 0.1^{\mathrm{a}}$ & ngd & ngd & ngd \\
\hline GAL401 & ngd & $0.83 \pm 0.15^{\mathrm{d}}$ & $2.68 \pm 0.35^{c}$ & $6.19 \pm 0.25^{\mathrm{b}}$ & $8.76 \pm 0.23^{\mathrm{a}}$ & ngd & ngd & ngd \\
\hline GCL642 & ngd & $3.39 \pm 0.09^{\mathrm{d}}$ & $5.95 \pm 0.14^{\mathrm{b}}$ & $7.28 \pm 0.20^{\mathrm{a}}$ & $4.21 \pm 0.21^{\mathrm{c}}$ & ngd & ngd & ngd \\
\hline GCL448 & ngd & $1.89 \pm 0.17^{\mathrm{d}}$ & $5.18 \pm 0.15^{\mathrm{c}}$ & $8.57 \pm 0.18^{\mathrm{a}}$ & $6.93 \pm 0.10^{\mathrm{b}}$ & ngd & ngd & ngd \\
\hline GPL336 & ngd & $1.12 \pm 0.12^{c}$ & $2.64 \pm 0.01^{\mathrm{b}}$ & $4.21 \pm 0.07^{\mathrm{a}}$ & $0.45 \pm 0.05^{\mathrm{d}}$ & ngd & ngd & ngd \\
\hline GRL334 & ngd & $2.96 \pm 0.07^{\mathrm{c}}$ & $6.26 \pm 0.10^{\mathrm{b}}$ & $8.41 \pm 0.32^{\mathrm{a}}$ & $7.99 \pm 0.14^{\mathrm{a}}$ & ngd & ngd & ngd \\
\hline GRL403 & ngd & $2.43 \pm 0.13^{\mathrm{d}}$ & $6.43 \pm 0.06^{c}$ & $8.96 \pm 0.12^{\mathrm{a}}$ & $8.29 \pm 0.17^{\mathrm{b}}$ & ngd & ngd & ngd \\
\hline GRC604 & ngd & $1.49 \pm 0.11^{c}$ & $3.48 \pm 0.11^{\mathrm{b}}$ & $5.59 \pm 0.02^{\mathrm{a}}$ & $5.53 \pm 0.07^{\mathrm{a}}$ & ngd & ngd & ngd \\
\hline GRS805 & ngd & $0.41 \pm 0.03^{\mathrm{b}}$ & $3.28 \pm 0.22^{\mathrm{a}}$ & $3.63 \pm 0.45^{\mathrm{a}}$ & ngd & ngd & ngd & ngd \\
\hline HCC522 & ngd & $8.93 \pm 0.03^{\mathrm{e}}$ & $17.36 \pm 0.77^{\mathrm{d}}$ & $24.61 \pm 0.47^{\mathcal{C}}$ & $32.63 \pm 0.58^{\mathrm{a}}$ & $32.41 \pm 0.47^{\mathrm{a}}$ & $28.63 \pm 0.12^{b}$ & \\
\hline HEL801 & ngd & $6.89 \pm 0.16^{c}$ & $9.69 \pm 0.18^{\mathrm{b}}$ & $13.17 \pm 0.07^{\mathrm{a}}$ & $12.25 \pm 0.78^{\mathrm{a}}$ & ngd & ngd & ngd \\
\hline HEL802 & ngd & $0.10 \pm 0.05^{\mathrm{b}}$ & $0.43 \pm 0.06^{\mathrm{b}}$ & $1.22 \pm 0.27^{\mathrm{a}}$ & $1.46 \pm 0.11^{\mathrm{a}}$ & ngd & ngd & ngd \\
\hline HAL340 & ngd & $2.79 \pm 0.13^{c}$ & $6.46 \pm 0.20^{\mathrm{b}}$ & $8.29 \pm 0.07^{\mathrm{a}}$ & $8.48 \pm 0.08^{\mathrm{a}}$ & ngd & ngd & \\
\hline HUL417 & ngd & $1.03 \pm 0.13^{\mathrm{c}}$ & $2.21 \pm 0.04^{\mathrm{a}}$ & $1.49 \pm 0.31^{\mathrm{b}}$ & $0.08 \pm 0.03^{\mathrm{d}}$ & ngd & ngd & ngd \\
\hline ITL314 & ngd & $0.46 \pm 0.00^{\mathrm{d}}$ & $2.92 \pm 0.18^{\mathrm{c}}$ & $4.44 \pm 0.96^{\mathrm{b}}$ & $4.96 \pm 0.08^{\mathrm{a}}$ & $3.05 \pm 0.08^{c}$ & ngd & ngd \\
\hline ILC238 & ngd & $4.20 \pm 0.06^{\mathrm{d}}$ & $7.65 \pm 0.24^{c}$ & $10.83 \pm 0.24^{\mathrm{b}}$ & $12.19 \pm 0.27^{\mathrm{a}}$ & $2.51 \pm 0.10^{\mathrm{e}}$ & ngd & ngd \\
\hline LSL331 & ngd & $3.78 \pm 0.23^{c}$ & $5.79 \pm 0.05^{\mathrm{b}}$ & $7.92 \pm 0.17^{\mathrm{a}}$ & $7.84 \pm 0.24^{\mathrm{a}}$ & ngd & ngd & ngd \\
\hline LSL332 & ngd & $1.17 \pm 0.24^{\mathrm{d}}$ & $3.53 \pm 0.16^{\mathrm{c}}$ & $5.73 \pm 0.17^{\mathrm{b}}$ & $7.84 \pm 0.28^{\mathrm{a}}$ & ngd & ngd & ngd \\
\hline LES812 & ngd & $1.97 \pm 0.16^{\mathrm{b}}$ & $3.07 \pm 0.16^{\mathrm{a}}$ & $2.80 \pm 0.09^{\mathrm{a}}$ & $0.44 \pm 0.05^{\mathrm{c}}$ & ngd & ngd & ngd \\
\hline NLL317 & ngd & $1.05 \pm 0.09^{c}$ & $3.03 \pm 0.07^{\mathrm{b}}$ & $4.35 \pm 0.17^{\mathrm{a}}$ & $4.17 \pm 0.14^{\mathrm{a}}$ & ngd & ngd & ngd \\
\hline OIL347 & ngd & $1.60 \pm 0.06^{c}$ & $3.55 \pm 0.08^{\mathrm{a}}$ & $2.85 \pm 0.13^{\mathrm{b}}$ & $1.49 \pm 0.09^{c}$ & ngd & ngd & \\
\hline PFL346 & ngd & $1.37 \pm 0.10^{\mathrm{d}}$ & $3.08 \pm 0.04^{c}$ & $4.95 \pm 0.13^{\mathrm{a}}$ & $3.67 \pm 0.08^{b}$ & ngd & ngd & \\
\hline PHL322 & ngd & $8.18 \pm 0.23^{\mathrm{d}}$ & $21.36 \pm 0.24^{\mathrm{c}}$ & $29.28 \pm 0.20^{\mathrm{b}}$ & $36.64 \pm 0.91^{\mathrm{a}}$ & $35.94 \pm 0.66^{\mathrm{a}}$ & $35.61 \pm 0.20^{\mathrm{a}}$ & ngd \\
\hline PHC571 & ngd & $9.89 \pm 0.12^{\mathrm{f}}$ & $22.98 \pm 0.16^{\mathrm{e}}$ & $30.46 \pm 0.90^{c}$ & $34.23 \pm 0.30^{\mathrm{b}}$ & $40.30 \pm 0.09^{a}$ & $26.21 \pm 0.62^{\mathrm{d}}$ & ngd \\
\hline PNS806 & ngd & $2.69 \pm 0.22^{c}$ & $4.39 \pm 0.29^{\mathrm{a}}$ & $3.35 \pm 0.20^{\mathrm{b}}$ & $0.26 \pm 0.09^{\mathrm{d}}$ & ngd & ngd & ngd \\
\hline PAC891 & $0.22 \pm 0.04^{\mathrm{b}}$ & $0.34 \pm 0.01^{\mathrm{b}}$ & $1.17 \pm 0.27^{\mathrm{a}}$ & $1.47 \pm 0.31^{\mathrm{a}}$ & $0.38 \pm 0.29^{b}$ & ngd & ngd & ngd \\
\hline PAC854 & $0.10 \pm 0.02^{\mathrm{b}}$ & $0.26 \pm 0.11^{\mathrm{b}}$ & $1.02 \pm 0.23^{\mathrm{a}}$ & $0.69 \pm 0.13^{\mathrm{a}}$ & $0.09 \pm 0.05^{\mathrm{b}}$ & ngd & ngd & ngd \\
\hline PCC884 & $0.47 \pm 0.01^{c}$ & $1.58 \pm 0.07^{\mathrm{b}}$ & $2.62 \pm 0.59^{\mathrm{ab}}$ & $3.72 \pm 0.95^{\mathrm{a}}$ & $3.50 \pm 0.37^{\mathrm{a}}$ & ngd & ngd & ngd \\
\hline PCL660 & $0.85 \pm 0.03^{c}$ & $1.05 \pm 0.02^{c}$ & $3.34 \pm 0.30^{\mathrm{b}}$ & $5.07 \pm 0.27^{\mathrm{a}}$ & $3.59 \pm 0.10^{\mathrm{b}}$ & ngd & ngd & ngd \\
\hline PCC897 & ngd & $0.24 \pm 0.08^{c}$ & $1.54 \pm 0.45^{\mathrm{b}}$ & $2.08 \pm 0.25^{\mathrm{b}}$ & $2.85 \pm 0.10^{\mathrm{a}}$ & $0.58 \pm 0.07^{c}$ & ngd & ngd \\
\hline PDC855 & $0.40 \pm 0.06^{c}$ & $0.90 \pm 0.08^{c}$ & $4.17 \pm 0.22^{\mathrm{b}}$ & $7.09 \pm 0.60^{\mathrm{a}}$ & $6.33 \pm 0.29^{\mathrm{a}}$ & ngd & ngd & ngd \\
\hline PEC847 & $0.22 \pm 0.02^{\mathrm{c}}$ & $0.57 \pm 0.10^{c}$ & $2.72 \pm 0.33^{\mathrm{b}}$ & $4.67 \pm 0.18^{\mathrm{a}}$ & $2.85 \pm 0.01^{\mathrm{b}}$ & ngd & ngd & ngd \\
\hline PEL063 & ngd & $0.38 \pm 0.05^{\mathrm{d}}$ & $1.60 \pm 0.00^{c}$ & $2.96 \pm 0.31^{\mathrm{a}}$ & $2.51 \pm 0.20^{\mathrm{b}}$ & ngd & ngd & ngd \\
\hline PEC810 & $0.29 \pm 0.04^{c}$ & $0.70 \pm 0.07^{\mathrm{c}}$ & $2.10 \pm 0.10^{\mathrm{b}}$ & $3.15 \pm 0.37^{\mathrm{a}}$ & $2.31 \pm 0.14^{\mathrm{b}}$ & ngd & ngd & ngd \\
\hline PEC856 & $0.60 \pm 0.04^{c}$ & $1.28 \pm 0.14^{\mathrm{b}}$ & $4.16 \pm 0.43^{\mathrm{a}}$ & $4.54 \pm 0.12^{\mathrm{a}}$ & $0.41 \pm 0.11^{\mathrm{c}}$ & ngd & ngd & ngd \\
\hline PFC860 & $0.56 \pm 0.06^{\mathrm{b}}$ & $1.02 \pm 0.11^{\mathrm{ab}}$ & $1.32 \pm 0.08^{\mathrm{a}}$ & $1.15 \pm 0.38^{\mathrm{a}}$ & $0.09 \pm 0.00^{c}$ & ngd & ngd & ngd \\
\hline
\end{tabular}


TABLE 2: Continued.

\begin{tabular}{|c|c|c|c|c|c|c|c|c|}
\hline \multirow{2}{*}{ Strain } & \multicolumn{8}{|c|}{ Temperature } \\
\hline & 12 & 17 & 22 & 27 & 32 & 37 & 42 & 47 \\
\hline PNL126 & $0.21 \pm 0.06^{c}$ & $0.45 \pm 0.04^{\mathrm{c}}$ & $1.39 \pm 0.24^{\mathrm{a}}$ & $1.04 \pm 0.11^{\mathrm{b}}$ & $0.13 \pm 0.02^{c}$ & ngd & ngd & ngd \\
\hline PNC816 & $0.22 \pm 0.03^{\mathrm{bc}}$ & $0.56 \pm 0.08^{\mathrm{b}}$ & $1.12 \pm 0.42^{\mathrm{a}}$ & $0.46 \pm 0.06^{\mathrm{b}}$ & $0.03 \pm 0.02^{c}$ & ngd & ngd & ngd \\
\hline POC 843 & $0.77 \pm 0.31^{\mathrm{c}}$ & $2.33 \pm 0.13^{b c}$ & $4.42 \pm 0.38^{\mathrm{ab}}$ & $5.89 \pm 0.20^{\mathrm{a}}$ & $4.33 \pm 0.06^{\mathrm{ab}}$ & ngd & ngd & ngd \\
\hline POC 847 & $0.68 \pm 0.10^{\mathrm{cd}}$ & $1.46 \pm 0.24^{\mathrm{bc}}$ & $6.43 \pm 0.19^{\mathrm{a}}$ & $7.28 \pm 0.16^{\mathrm{a}}$ & $1.80 \pm 0.74^{\mathrm{b}}$ & $0.26 \pm 0.00^{\mathrm{d}}$ & ngd & ngd \\
\hline POL067 & $1.33 \pm 0.25^{\mathrm{d}}$ & $2.64 \pm 0.05^{\mathrm{c}}$ & $7.26 \pm 0.08^{\mathrm{a}}$ & $7.36 \pm 0.43^{\mathrm{a}}$ & $4.00 \pm 0.63^{\mathrm{b}}$ & ngd & ngd & ngd \\
\hline PPL111 & $0.66 \pm 0.08^{\mathrm{d}}$ & $1.06 \pm 0.35^{\mathrm{d}}$ & $4.09 \pm 0.25^{\mathrm{c}}$ & $7.01 \pm 0.15^{\mathrm{a}}$ & $6.24 \pm 0.26^{\mathrm{b}}$ & ngd & ngd & ngd \\
\hline PPC823 & $0.70 \pm 0.05^{\mathrm{b}}$ & $0.91 \pm 0.23^{\mathrm{b}}$ & $3.37 \pm 0.40^{\mathrm{a}}$ & $3.36 \pm 0.82^{\mathrm{a}}$ & $0.94 \pm 0.09^{b}$ & ngd & ngd & ngd \\
\hline PSC757 & $0.64 \pm 0.22^{\mathrm{bc}}$ & $1.07 \pm 0.36^{\mathrm{ab}}$ & $1.35 \pm 0.08^{\mathrm{a}}$ & $1.18 \pm 0.14^{\mathrm{a}}$ & $0.43 \pm 0.01^{\mathrm{cd}}$ & ngd & ngd & ngd \\
\hline PTC822 & $0.36 \pm 0.07^{\mathrm{c}}$ & $0.54 \pm 0.10^{c}$ & $1.54 \pm 0.47^{\mathrm{b}}$ & $3.35 \pm 0.50^{\mathrm{a}}$ & $2.01 \pm 0.02^{\mathrm{b}}$ & ngd & ngd & ngd \\
\hline SHC608 & ngd & $7.73 \pm 0.15^{\mathrm{c}}$ & $12.28 \pm 0.16^{\mathrm{a}}$ & $12.29 \pm 0.11^{\mathrm{a}}$ & $8.37 \pm 0.24^{\mathrm{b}}$ & $0.21 \pm 0.00^{\mathrm{d}}$ & ngd & ngd \\
\hline THC610 & ngd & $3.76 \pm 0.23^{\mathrm{e}}$ & $6.90 \pm 0.10^{c}$ & $8.47 \pm 0.07^{\mathrm{b}}$ & $9.66 \pm 0.42^{\mathrm{a}}$ & $5.44 \pm 0.11^{\mathrm{d}}$ & ngd & ngd \\
\hline TLL473 & ngd & $2.17 \pm 0.06^{\mathrm{e}}$ & $4.55 \pm 0.05^{\mathrm{d}}$ & $6.55 \pm 0.10^{c}$ & $8.01 \pm 0.16^{\mathrm{a}}$ & $7.47 \pm 0.05^{\mathrm{b}}$ & ngd & ngd \\
\hline TVL469 & ngd & $4.60 \pm 0.09^{\mathrm{d}}$ & $6.89 \pm 0.14^{c}$ & $8.49 \pm 0.14^{\mathrm{b}}$ & $8.88 \pm 0.13^{\mathrm{a}}$ & $0.38 \pm 0.10^{\mathrm{e}}$ & ngd & ngd \\
\hline TVC614 & ngd & $5.39 \pm 0.49^{c}$ & $10.53 \pm 0.18^{\mathrm{a}}$ & $11.24 \pm 0.23^{\mathrm{a}}$ & $10.93 \pm 0.58^{\mathrm{a}}$ & $1.52 \pm 0.16^{\mathrm{b}}$ & ngd & ngd \\
\hline TLC616 & ngd & $5.55 \pm 0.17^{\mathrm{d}}$ & $8.01 \pm 0.12^{c}$ & $9.29 \pm 0.06^{\mathrm{b}}$ & $10.25 \pm 0.12^{\mathrm{a}}$ & $0.48 \pm 0.02^{\mathrm{e}}$ & ngd & ngd \\
\hline
\end{tabular}

As anticipated, mycelium growth on PDA was in all cases significantly higher than on OMW-based solidified media with the only exception of Ganoderma carnosum GCL448 growing on 25\% OMW. Still, several other strains presented satisfactory growth on both $25 \%$ and $50 \%$ OMW, while some performed adequately on 100\% OMW as well. Such noteworthy cases included (apart from GCL448) Abortiporus biennis, Coriolopsis trogii, Daedalea quercina, Ganoderma adspersum, G. resinaceum (GRL344 and GRL403), Hapalopilus croceus, Hericium erinaceus (HEL801 only), Irpex lacteus, Phanerochaete chrysosporium, Pleurotus djamor, P. pulmonarius, Stereum hirsutum, Trametes versicolor, and Tyromyces lacteus. On the other hand, practically no growth was detected on OMW-based media for Auricularia mesenterica, Fistulina hepatica, Grifola frondosa, Hericium erinaceus (HEL802 only), Hypsizygus ulmarius, Laetiporus sulphureus (LSL331 only), Lentinula edodes, Pholiota nameko, and Pleurotus abieticola. In some of the rather few cases in which a species was represented by more than one individual, results occasionally revealed much different behavior between certain conspecific strains, for example, in Ganoderma carnosum, Hericium erinaceus, and Laetiporus sulphureus. This observation is in accordance with previous findings reporting high intraspecific variability within Pleurotus spp. in the degradation of OMW phenolics [32], which advocates for a strain (rather than a species) dependent behavior.

Consequently, eight fungi (i.e., Abortiporus biennis ABL436, Ganoderma carnosum GCL448, Hapalopilus croceus HCC522, Hericium erinaceus HEL801, Irpex lacteus ILC238, Phanerochaete chrysosporium PHL322, Pleurotus djamor PDC855, and P. pulmonarius PPL111) were qualified for further study on the basis of the outcome of the previous experiment, on the effect their growth had on the decolorization of the OMW-based substrate (assessed by visual inspection, data not shown), and on the prospects that individual species present in their subsequent/future exploitation for the generation of value-added products (e.g., edible biomass from Pleurotus spp.).
In all cases, fungal growth resulted in $\mathrm{pH}$ decrease from an initial value of 5.65 to values of as low as 4.48 for I. lacteus, while for the rest of the strains it ranged from 5.00 to 5.50 (Table 4). However, differences in $\mathrm{pH}$ were less pronounced for Pleurotus spp., which is in accordance with previous pertinent results obtained after the use of $P$. ostreatus [39]. On the other hand, electric conductivity measurements demonstrated a gradual increase from $1.80 \mathrm{mS} \mathrm{cm}^{-1}$ in the initial material to $3.85-5.97 \mathrm{mS} \mathrm{cm}^{-1}$ in the biodegraded effluent (Table 4); this seems to be the general trend for conductivity values after microbial treatment of raw OMW [40].

All selected strains produced abundant mycelium in static batch cultures; yet four out of the eight strains (A. biennis, $H$. croceus, $P$. djamor, and $P$. pulmonarius) provided significantly higher yields (200-230 mg) than the rest (Table 4). Nevertheless, biomass production at initial growth stages was rather poor since substrate's content in readily assimilated nutrients was low [2]. In general, fungal growth was accompanied with varying degrees of enzymes production among the various strains examined. A. biennis was the only fungus to demonstrate a particularly high laccase activity within the initial cultivation period; that is, a peak value of $205 \mathrm{U} \mathrm{L}^{-1}$ was obtained after 10 days only, and then it subsided and remained rather constant at values of $115-122 \mathrm{U} \mathrm{L}^{-1}$ until the end of the cultivation (Figure 2(a)). The rest of the strains demonstrated either significantly lower laccase activities or no activities at all. Within the former group, laccase production for both Pleurotus spp. and G. carnosum increased in parallel with biomass growth and reached the highest value by the end of cultivation (63-79 $\left.\mathrm{UL}^{-1}\right)$, whereas $H$. croceus and $P h$. chrysosporium presented much lower values $\left(3.6-5.1 \mathrm{UL}^{-1}\right)$. In contrast, in the cases of $H$. erinaceus and I. lacteus no laccase activity was detected.

As regards $\mathrm{Mn}$ peroxidase $(\mathrm{MnP})$, a large variability was detected among strains in terms of both the peak activity values and the time periods where these were produced (Figure 2(b)). Hence, the highest $\mathrm{MnP}$ activities were observed for 
TABLE 3: Mycelium linear growth rates $\left(K_{r}, \mathrm{~mm} \mathrm{day}^{-1}\right)$ for 60 strains of macrofungi growing on PDA and on OMW-based substrates prepared in various dilutions in water $(\mathrm{v} / \mathrm{v})$; ngd: no growth detected. Values are expressed as means \pm standard deviation of means, $n=3$. Lack of common superscripts indicates statistically significant differences (Gabriel's $t$-test, $P<0.05$ ) for comparisons of treatment means between different strains (numbers) and different substrates (lowercase letters).

\begin{tabular}{|c|c|c|c|c|}
\hline \multirow{2}{*}{ Strain } & \multicolumn{4}{|c|}{ Substrate } \\
\hline & PDA & 25\% OMW & 50\% OMW & $100 \%$ OMW \\
\hline ABC521 & $10.56 \pm 0.09^{5, a}$ & $6.31 \pm 0.15^{6,7,8, b}$ & $4.59 \pm 0.08^{6,7,8 c}$ & $2.64 \pm 0.05^{3,4, \mathrm{~d}}$ \\
\hline ABL436 & $11.86 \pm 0.17^{4, \mathrm{a}}$ & $9.03 \pm 0.10^{4,5, \mathrm{~b}}$ & $7.65 \pm 0.28^{1 \mathrm{c}}$ & $5.15 \pm 0.05^{1, \mathrm{~d}}$ \\
\hline ACL834 & $3.81 \pm 0.38^{16,17,18,19, \mathrm{a}}$ & $2.38 \pm 0.13^{15,16, \mathrm{~b}}$ & $1.81 \pm 0.19^{13,14, \mathrm{~b}}$ & ngd \\
\hline AML472 & $4.88 \pm 1.00^{13,14,15,16, \mathrm{a}}$ & ngd & ngd & ngd \\
\hline CTL447 & $9.20 \pm 0.16^{6 a}$ & $6.84 \pm 0.06^{6, b}$ & $4.16 \pm 0.10^{7,8,9 c}$ & $2.40 \pm 0.13^{4,5, \mathrm{~d}}$ \\
\hline DQC528 & $10.96 \pm 0.10^{5, \mathrm{a}}$ & $6.04 \pm 0.06^{7,8,9, \mathrm{~b}}$ & $5.94 \pm 0.08^{3,4 b}$ & $5.23 \pm 0.18^{1 \mathrm{c}}$ \\
\hline DSC750 & $11.19 \pm 0.46^{4,5, \mathrm{a}}$ & $2.87 \pm 0.04^{14,15, \mathrm{~b}}$ & ngd & ngd \\
\hline FHL295 & $1.88 \pm 0.12^{24,25,26,27, a}$ & ngd & ngd & ngd \\
\hline FVS803 & $5.71 \pm 0.34^{11,12,13, \mathrm{a}}$ & $3.98 \pm 0.19^{12,13, \mathrm{~b}}$ & $2.88 \pm 0.26^{11 \mathrm{c}}$ & ngd \\
\hline FPL302 & $8.04 \pm 0.14^{7,8,9, \mathrm{a}}$ & $0.40 \pm 0.00^{22,23,24, \mathrm{~b}}$ & ngd & ngd \\
\hline GAL401 & $9.03 \pm 0.20^{6,7, \mathrm{a}}$ & $6.05 \pm 0.29^{6,7,8,9, \mathrm{~b}}$ & $3.88 \pm 0.18^{9, \mathrm{c}}$ & $2.51 \pm 0.12^{4, \mathrm{~d}}$ \\
\hline GCL642 & $6.20 \pm 0.19^{11,12, \mathrm{a}}$ & $2.44 \pm 0.33^{15,16, \mathrm{~b}}$ & $1.70 \pm 0.13^{13,14, \mathrm{c}}$ & ngd \\
\hline GCL448 & $7.52 \pm 0.18^{9,10, \mathrm{a}}$ & $6.62 \pm 0.17^{6,7 \mathrm{a}}$ & $5.45 \pm 0.62^{4,5 b}$ & $2.29 \pm 0.09^{4,5 c}$ \\
\hline GPL336 & $2.57 \pm 0.23^{22,23,24,25, \mathrm{a}}$ & $1.68 \pm 0.06^{16,17,18 b}$ & $0.90 \pm 0.03^{16,17,18,19 c}$ & $0.54 \pm 0.06^{7,8,9,10, \mathrm{~d}}$ \\
\hline GRL344 & $7.73 \pm 0.42^{8,9, \mathrm{a}}$ & $5.43 \pm 0.91^{9,10, \mathrm{~b}}$ & $4.83 \pm 0.33^{5,6, \mathrm{~b}}$ & $2.32 \pm 0.49^{4,5, \mathrm{c}}$ \\
\hline GRL403 & $8.12 \pm 0.03^{7,8,9, \mathrm{a}}$ & $5.87 \pm 0.00^{7,8,9, \mathrm{~b}}$ & $3.96 \pm 0.06^{8,9, \mathrm{c}}$ & $2.14 \pm 0.14^{4,5, \mathrm{~d}}$ \\
\hline GRC604 & $4.62 \pm 0.10^{14,15,16,17, \mathrm{a}}$ & $3.57 \pm 0.08^{13,14, \mathrm{~b}}$ & $2.59 \pm 0.18^{11,12 c}$ & $0.96 \pm 0.15^{6,7 \mathrm{~d}}$ \\
\hline GFS805 & $3.24 \pm 0.19^{17,18,19,20, \mathrm{a}}$ & ngd & ngd & ngd \\
\hline HCC522 & $32.99 \pm 0.57^{1, \mathrm{a}}$ & $12.12 \pm 0.10^{1, \mathrm{~b}}$ & $1.05 \pm 0.00^{15,16,17,18 c}$ & ngd \\
\hline HEL801 & $13.96 \pm 0.07^{2, a}$ & $8.27 \pm 0.89^{5, \mathrm{~b}}$ & $5.51 \pm 0.21^{4 \mathrm{c}}$ & $3.16 \pm 0.12^{2,3 \mathrm{~d}}$ \\
\hline HEL802 & $1.50 \pm 0.28^{25,26,27,28, \mathrm{a}}$ & ngd & ngd & ngd \\
\hline HAL340 & $7.58 \pm 0.04^{8,9,10, \mathrm{a}}$ & $3.62 \pm 0.13^{13,14, \mathrm{~b}}$ & $2.08 \pm 0.09^{12,13 c}$ & $0.98 \pm 0.03^{6,7 \mathrm{~d}}$ \\
\hline HUL417 & $1.87 \pm 0.02^{24,25,26,27, \mathrm{a}}$ & ngd & ngd & ngd \\
\hline ITL314 & $4.45 \pm 0.04^{14,15,16,17, \mathrm{a}}$ & $0.08 \pm 0.03^{24, \mathrm{~b}}$ & ngd & ngd \\
\hline ILC238 & $13.42 \pm 0.50^{2,3, \mathrm{a}}$ & $10.04 \pm 0.05^{2,3, \mathrm{~b}}$ & $6.80 \pm 0.22^{2, \mathrm{c}}$ & $0.70 \pm 0.00^{7,8,9, \mathrm{~d}}$ \\
\hline LSL331 & $4.19 \pm 0.79^{15,16,17,18, \mathrm{a}}$ & ngd & ngd & ngd \\
\hline LSL332 & $6.53 \pm 0.48^{10,11, \mathrm{a}}$ & $2.86 \pm 0.14^{14,15, \mathrm{~b}}$ & $2.71 \pm 0.08^{11,12, \mathrm{~b}, \mathrm{c}}$ & $1.96 \pm 0.33^{5, c}$ \\
\hline LES812 & $2.33 \pm 0.20^{23,24,25,26, a}$ & ngd & ngd & ngd \\
\hline NLL317 & $3.95 \pm 0.22^{16,17,18,19 a}$ & $1.70 \pm 0.34^{16,17,18 b}$ & ngd & ngd \\
\hline OIL347 & $2.25 \pm 0.05^{23,24,25,26, a}$ & $1.49 \pm 0.04^{17,18,19, \mathrm{~b}}$ & $0.80 \pm 0.00^{16,17,18,19, c}$ & $0.42 \pm 0.08^{8,9,10, \mathrm{~d}}$ \\
\hline PFL346 & $3.22 \pm 0.05^{20,21,22,23, \mathrm{a}}$ & $2.09 \pm 0.04^{15,16,17, \mathrm{~b}}$ & $1.35 \pm 0.03^{14,15,16, \mathrm{c}}$ & $0.55 \pm 0.03^{7,8,9,10, \mathrm{~d}}$ \\
\hline PHL322 & $36.64 \pm 0.92^{1, a}$ & $12.57 \pm 0.18^{1, \mathrm{~b}}$ & $6.48 \pm 0.03^{2,3 c}$ & ngd \\
\hline PHC571 & $34.23 \pm 0.30^{1 \mathrm{a}}$ & $10.83 \pm 0.10^{2, b}$ & $4.18 \pm 0.15^{6,7,8,9, \mathrm{c}}$ & ngd \\
\hline PNS806 & $3.97 \pm 0.38^{16,17,18,19, \mathrm{a}}$ & ngd & ngd & ngd \\
\hline PAC891 & $2.74 \pm 0.11^{21,22,23,24, \mathrm{a}}$ & $0.44 \pm 0.16^{22,23,24, \mathrm{~b}}$ & ngd & ngd \\
\hline PAC854 & $1.02 \pm 0.12^{\mathrm{a}}$ & ngd & ngd & ngd \\
\hline PCC 884 & $4.69 \pm 0.05^{13,14,15,16, \mathrm{a}}$ & $2.56 \pm 0.32^{15, \mathrm{~b}}$ & $2.06 \pm 0.17^{14,15,16,17, \mathrm{~b}}$ & $0.23 \pm 0.05^{9,10, \mathrm{c}}$ \\
\hline PCL660 & $2.55 \pm 0.68^{22,23,24,25, \mathrm{a}}$ & $0.06 \pm 0.01^{22,23,24, \mathrm{~b}}$ & ngd & ngd \\
\hline PCC897 & $2.30 \pm 0.23^{23,24,25,26, a}$ & $1.05 \pm 0.12^{18,19,20,21 b}$ & $0.58 \pm 0.16^{17,18,19,20 c}$ & ngd \\
\hline PDC855 & $5.38 \pm 0.28^{12,13,14, \mathrm{a}}$ & $4.19 \pm 0.29^{11,12,13, \mathrm{~b}}$ & $3.09 \pm 0.27^{10,11, c}$ & $0.78 \pm 0.24^{6,7,8, \mathrm{~d}}$ \\
\hline PEC847 & $3.98 \pm 0.38^{16,17,18,19, \mathrm{a}}$ & $0.87 \pm 0.06^{20,21,22,23, \mathrm{~b}}$ & $0.32 \pm 0.03^{19,20,21, \mathrm{c}}$ & ngd \\
\hline PEL063 & $3.59 \pm 0.24^{18,19,20,21, \mathrm{a}}$ & $0.90 \pm 0.09^{19,20,21,22, \mathrm{~b}}$ & $0.20 \pm 0,00^{20,21, \mathrm{c}}$ & ngd \\
\hline PEC810 & $3.76 \pm 0.12^{17,18,19,20, \mathrm{a}}$ & $1.30 \pm 0,34^{17,18,19,20, \mathrm{~b}}$ & $0.42 \pm 0.13^{18,19,20, \mathrm{c}}$ & ngd \\
\hline PEC856 & $1.48 \pm 0.04^{26,27,28, \mathrm{a}}$ & $0.42 \pm 0.20^{22,23,24, \mathrm{~b}}$ & ngd & ngd \\
\hline PFC860 & $1.03 \pm 0.28^{27,28, \mathrm{a}}$ & $0.51 \pm 0.02^{21,22,23,24, \mathrm{~b}}$ & $0.32 \pm 0.03^{19,20,21, \mathrm{~b}}$ & ngd \\
\hline
\end{tabular}


TABLE 3: Continued.

\begin{tabular}{|c|c|c|c|c|}
\hline \multirow{2}{*}{ Strain } & \multicolumn{4}{|c|}{ Substrate } \\
\hline & PDA & $25 \%$ OMW & $50 \%$ OMW & $100 \%$ OMW \\
\hline PNL126 & $0.94 \pm 0.01^{27,28, \mathrm{a}}$ & $0.36 \pm 0.02^{23,24, \mathrm{~b}}$ & $0.07 \pm 0.04^{21, c}$ & ngd \\
\hline PNC816 & $0.64 \pm 0.04^{28, \mathrm{a}}$ & $0.15 \pm 0.10^{22,23,24, \mathrm{~b}}$ & ngd & ngd \\
\hline POC 843 & $3.45 \pm 0.69^{19,20,21,22, a}$ & $1.74 \pm 0.06^{16,17,18, \mathrm{~b}}$ & $0.47 \pm 0.41^{18,19,20, c}$ & ngd \\
\hline POC847 & $5.10 \pm 0.03^{12,13,14, \mathrm{a}}$ & $1.76 \pm 0.07^{16,17,18, \mathrm{~b}}$ & $1.15 \pm 0.05^{18,19,20,21, \mathrm{c}}$ & ngd \\
\hline POL067 & $6.64 \pm 0.22^{10,11, \mathrm{a}}$ & $2.39 \pm 0.05^{15,16, \mathrm{~b}}$ & $0.75 \pm 0.48^{16,17,18,19, c}$ & $0.07 \pm 0.03^{10, c}$ \\
\hline PPL111 & $7.80 \pm 0.21^{8,9, \mathrm{a}}$ & $2.74 \pm 0.05^{15, \mathrm{~b}}$ & $1.67 \pm 0.16^{13,14,15, \mathrm{c}}$ & ngd \\
\hline PPC823 & $3.71 \pm 0.28^{17,18,19,20, \mathrm{a}}$ & $1.56 \pm 0.10^{17,18,19 b}$ & $1.06 \pm 0.09^{15,16,17,18, \mathrm{c}}$ & $0.20 \pm 0.00^{9,10, \mathrm{~d}}$ \\
\hline PSC757 & $0.80 \pm 0.06^{27,28, \mathrm{a}}$ & $0.50 \pm 0.06^{22,23,24, \mathrm{~b}}$ & $0.30 \pm 0.00^{19,20,21, \mathrm{c}}$ & ngd \\
\hline PTC822 & $2.70 \pm 0.24^{21,22,23,24, \mathrm{a}}$ & $0.97 \pm 0.12^{18,19,20,21 b}$ & ngd & ngd \\
\hline SHC608 & $12.83 \pm 0.14^{3, \mathrm{a}}$ & $5.81 \pm 0.07^{8,9, \mathrm{~b}}$ & $4.71 \pm 0.03^{6,7, c}$ & $3.61 \pm 0.08^{2, \mathrm{~d}}$ \\
\hline THC610 & $9.08 \pm 0.06^{6,7, a}$ & $5.78 \pm 0.05^{8,9, \mathrm{~b}}$ & $3.74 \pm 0.19^{9,10, \mathrm{c}}$ & $2.62 \pm 0.02^{4, \mathrm{~d}}$ \\
\hline TLL473 & $7.40 \pm 0.12^{9,10, \mathrm{a}}$ & $4.93 \pm 0.03^{10,11, \mathrm{~b}}$ & $4.80 \pm 0.00^{10,11, \mathrm{~b}}$ & $1.24 \pm 0.10^{6, \mathrm{c}}$ \\
\hline TVL469 & $8.64 \pm 0.18^{6,7,8, \mathrm{a}}$ & $5.61 \pm 0.03^{8,9,10, \mathrm{~b}}$ & $4.37 \pm 0.03^{6,7,8,9, \mathrm{c}}$ & $3.61 \pm 0.08^{2, \mathrm{~d}}$ \\
\hline TVC614 & $8.98 \pm 0.18^{6,7, \mathrm{a}}$ & $4.42 \pm 0.04^{11,12, \mathrm{~b}}$ & $4.14 \pm 0.05^{7,8,9, \mathrm{~b}}$ & $3.37 \pm 0.07^{2, \mathrm{c}}$ \\
\hline TLC616 & $13.41 \pm 0.20^{2,3, \mathrm{a}}$ & $9.34 \pm 0.10^{3,4, \mathrm{~b}}$ & $6.77 \pm 0.07^{2, \mathrm{c}}$ & $2.61 \pm 0.06^{4, \mathrm{~d}}$ \\
\hline
\end{tabular}

H. erinaceus and $G$. carnosum (18 and $11 \mathrm{UL}^{-1}$, resp.) at the end of their growth. A. biennis, $H$. croceus, and I. lacteus presented their peak MnP values at or near the middle of the cultivation period $\left(1.6-5.9 \mathrm{U} \mathrm{L}^{-1}\right)$. On the other hand, $P h$. chrysosporium and the two Pleurotus spp. were early $\mathrm{MnP}$ producers since their maximum production $\left(0.4-3.0 \mathrm{U} \mathrm{L}^{-1}\right)$ was noted within the first 12 days of growth, and then it gradually subsided to levels of no detection at the end of cultivation. Production of $\mathrm{MnP}$ was previously associated with secondary metabolism activated by lack of adequate nitrogen and/or carbon [41]. This was apparently not the case during the early stages of incubation of Ph. chrysosporium and Pleurotus spp. used in this study, but it might well be the case for the other five strains which presented their peak activities with a time delay. On the other hand, no $\mathrm{Mn}$ independent peroxidase (MnIP) activities were detected by the two Pleurotus spp. tested (Figure 2(c)). All the other strains produced their peak MnIP values towards the second half of their growth period starting from day 12 ( $H$. croceus, $2.3 \mathrm{U} \mathrm{L}^{-1}$ ) and ending at day 25 (Irpex lacteus and A. biennis, $32 \mathrm{UL}^{-1}$ and $7.1 \mathrm{UL}^{-1}$, resp.).

In general, monitoring of enzymes production with respect to fungal growth indicated that $P$. djamor and $P$. pulmonarius shared identical patterns for all three activities, and this was also the case for $H$. croceus and Ph. chrysosporium. On the other hand, A. biennis presented common production patterns with I. lacteus as regards $\mathrm{MnP}$ and MnIP and so did $G$. carnosum with $H$. erinaceus.

Although in some cases the findings of the present study are in line with previous literature reports on enzyme activities in relation to fungal growth, for example, $\mathrm{MnP}$ activity for Pleurotus and Ganoderma spp. [32, 39], different types of data were also obtained. For example, MnIP activities in Ganoderma spp. showed their peak production earlier during the growth period [32] in comparison to the findings of the present study. Furthermore, previous pertinent results on $P$. eryngii and $P$. ostreatus revealed late production of $\mathrm{MnP}$ [32], which is in contrast with the results of this work (albeit with different Pleurotus spp.). While differences in enzyme activity values among strains used in various investigations could be attributed to both experimental conditions (including initial substrate composition and dilution rate) and properties of the particular fungus used, variations in enzyme production with respect to time could be primarily explained by the latter factor (i.e., variability of the biological material employed).

Total phenolic content was markedly reduced by all strains; however, particularly impressive was the reduction achieved by $A$. biennis, which after only six days of incubation decreased the initial phenols by more than $50 \%$, while by the end of the experiment the respective figure reached $92 \%$ (Table 4 ). Very good performers (although significantly inferior than the first) were the two Pleurotus species $(71 \%-$ $76 \%$ of decrease), followed by I. lacteus and G. carnosum. The latter was the second fastest (after A. biennis) in reducing total phenols since a $49 \%$ decrease was measured after just 12 days of incubation. It is noteworthy that other Ganoderma species/strains were reported to present similar behavior as regards the time needed to decrease to a similar extent OMW's phenolics, that is, by $41-44 \%$ within a 10 -day incubation period and by reaching a total reduction exceeding $64 \%$ $[26,32]$. On the other hand, selected $P$. ostreatus and P. eryngii strains demonstrated a rather delayed phenols degradation which exceeded the $50 \%$ level only after the completion of the first half of growth [32] much like as it was observed for $P$. djamor and $P$. pulmonarius in the present study. In certain cases (e.g., for A. biennis, G. carnosum, and I. lacteus), a notable deceleration in the reduction rate of total phenols reduction was observed during the second half of the growth period, which could be attributed to the recalcitrance of certain phenolics remaining in culture (e.g., trans-cinnamic acids which are not degraded by laccase) [42].

Of special interest was that total phenol reduction was significantly correlated with laccase and $\mathrm{MnP}$ activities $\left(r^{2}\right.$ $=0.43$ and $r^{2}=0.71$, resp.) when data from all eight fungi 


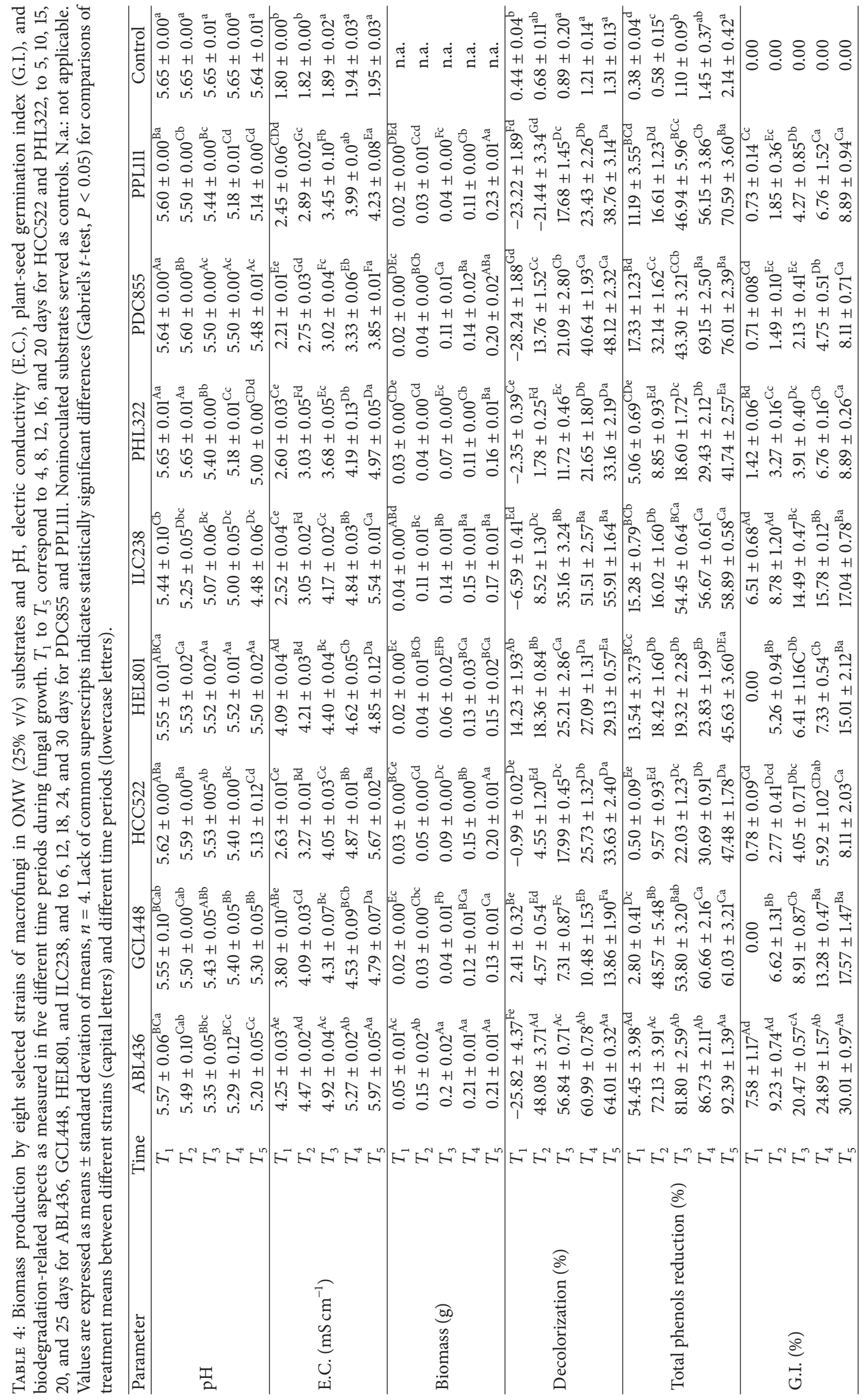




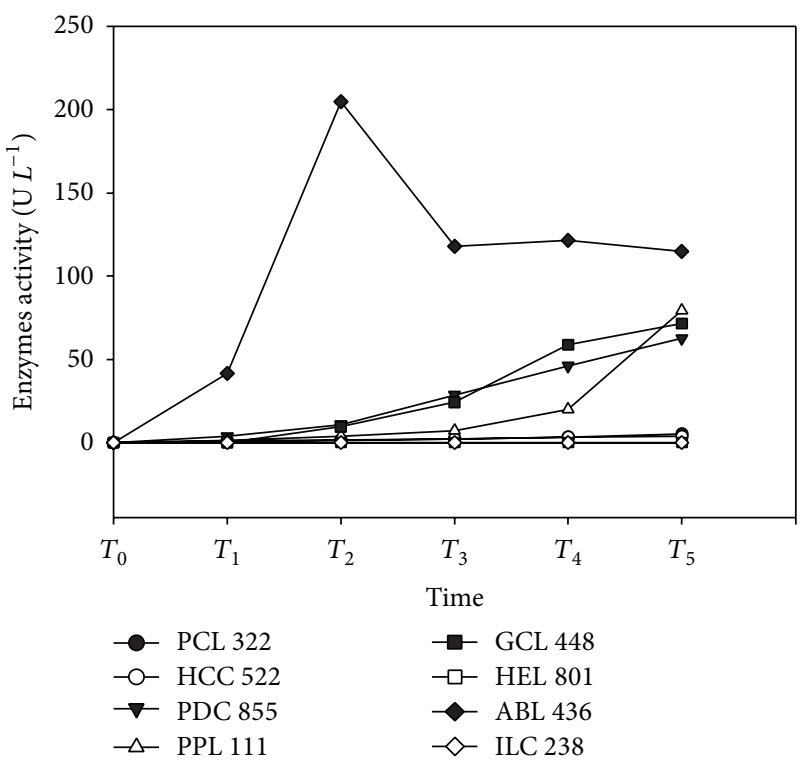

(a)

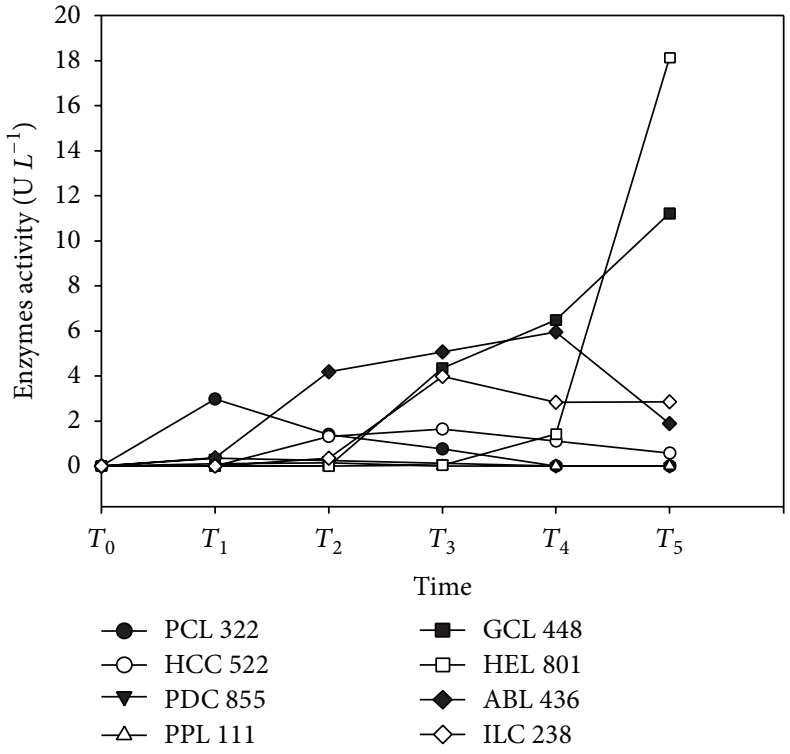

(b)

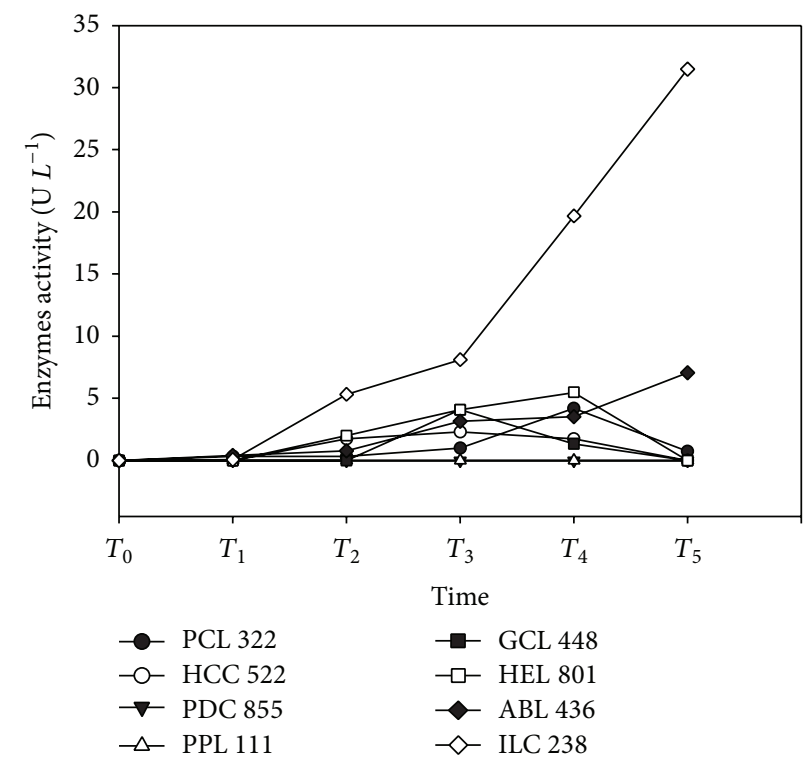

(c)

FIGURE 2: Enzyme activities ( $\mathrm{UL}^{-1}$ ) for laccase (a), manganese peroxidase, (b) and manganese-independent peroxidase (c) exhibited on OMW-based media ( $25 \% \mathrm{v} / \mathrm{v}$ in water) by eight selected macrofungi during a growth period of 20 to 30 days and in five different time points ( $T_{1}$ to $T_{5}$ ) as follows: 4, 8, 12, 16, and 20 days for H. croceus HCC522 and Ph. chrysosporium PHL322; 5, 10, 15, 20 , and 25 days for A. biennis ABL436, G. carnosum GCL448, H. erinaceus HEL801, and I. lacteus ILC238; 6, 12, 18, 24, and 30 days for P. djamor PDC855 and P. pulmonarius PPL111.

were collectively evaluated (Table 5), while these figures were found to be particularly high in the individual calculations made for $H$. croceus, Ph. chrysosporium, and Pleurotus spp. for laccase $\left(r^{2}=0.94-1.00\right)$, A. biennis, G. carnosum, $H$. erinaceus, and I. lacteus for $\mathrm{MnP}\left(r^{2}=0.71-0.94\right)$, and $A$. biennis, I. lacteus, and Ph. chrysosporium for MnIP $\left(r^{2}=\right.$ 0.81-1.00). Such correlations were detected not only during the biodegradation of OMW by $P$. ostreatus $[32,39]$ but also when effluents from the debittering of green olives were treated by several white-rot fungi [43]. In this category of microorganisms, production of laccase is often induced by the presence of the appropriate substrate in the growth environment [44]. Especially as regards OMW media, such induction for Pleurotus spp. was associated with the concentration of the effluent in the culture substrate; high laccase activities were detected when phenolics exceeded $1.5 \mathrm{~g} \mathrm{~L}^{-1}$ $[39,45,46]$, which is also the case with the initial phenolics content in the samples of this study $\left(>2.0 \mathrm{~g} \mathrm{~L}^{-1}\right)$. Most of the fungi examined here (with the only exception of A. biennis) demonstrated a lag phase for laccase production indicative of 
TABLE 5: Coefficient of determination $\left(r^{2}\right)$ values in comparison between all degradation parameters (biomass, total phenolics reduction, decolorization, and germination index) and enzyme activities on the basis of the entire dataset obtained from all eight macrofungi examined. ${ }^{*}$ Statistically significant at $5 \% ;{ }^{* *}$ statistically significant at $1 \%$.

\begin{tabular}{|c|c|c|c|c|c|c|c|}
\hline $\begin{array}{l}\text { OMW degradation } \\
\text { parameters and enzymes }\end{array}$ & $\begin{array}{c}\text { Fungal } \\
\text { biomass }\end{array}$ & $\begin{array}{l}\text { Laccase } \\
\text { activity }\end{array}$ & $\begin{array}{l}\text { Mn independent } \\
\text { peroxidase activity }\end{array}$ & $\begin{array}{l}\text { Mn peroxidase } \\
\text { activity }\end{array}$ & Decolorization & $\begin{array}{l}\text { Total phenolics } \\
\text { reduction }\end{array}$ & $\begin{array}{l}\text { Germination } \\
\text { index }\end{array}$ \\
\hline Fungal biomass & - & $0.50^{* *}$ & $0.56^{* *}$ & $0.56^{* *}$ & $0.86^{* *}$ & $0.76^{* *}$ & $0.88^{* *}$ \\
\hline Laccase activity & & - & 0.00 & 0.28 & $0.34^{*}$ & $0.63^{* *}$ & $0.51^{* *}$ \\
\hline $\begin{array}{l}\text { Mn independent } \\
\text { peroxidase activity }\end{array}$ & & & - & $0.55^{* *}$ & $0.46^{* *}$ & $0.32^{*}$ & $0.60^{* *}$ \\
\hline Mn peroxidase activity & & & & - & $0.33^{*}$ & $0.47^{* *}$ & $0.62^{* *}$ \\
\hline Decolorization & & & & & - & $0.71^{* *}$ & $0.71^{* *}$ \\
\hline Total phenolics reduction & & & & & & - & $0.84^{* *}$ \\
\hline Germination index & & & & & & & - \\
\hline
\end{tabular}

the time they needed to adapt their growth requirements to OMW [24, 47].

As previously noted with phenols content, A. biennis was the most efficient fungus in decolorizing OMW by reaching a $64 \%$ value by the end of the cultivation period (Table 4). During the process, color increased (for this and some other strains) during the first measurement(s) as a consequence of the oxidation and/or polycondensation of phenolic compounds in other darker-colored forms [44, 48]. Still, A. biennis achieved fast a very high decolorization rate ( $48 \%$ after 12 days) which is significantly higher than the respective values obtained from the other fungi during that same period (2\%-18\%). I. lacteus and Pleurotus spp. provided the next best total decolorization (56\% to $39 \%$ ), whereas $G$. carnosum performed rather poorly (14\%). On the other hand, $H$. erinaceus was the only fungus that performed significantly better during the first half of the growth period, and then its decolorization subsided considerably.

OMW decolorization efficacy by A. biennis is among the best reported in literature for basidiomycetes. In other pertinent studies, Pleurotus spp., Ganoderma applanatum, Lentinula edodes, Pycnoporus coccineus, Coriolopsis polyzona, and Lentinus tigrinus demonstrated similar magnitude of decolorization; however, in several of these cases initial medium and cultivation conditions varied considerably [26, $32,49,50]$. In addition, the outcome of this study demonstrates that decolorization is significantly correlated with total phenol reduction $\left(r^{2}=0.71\right)$, which is in line with previous results associating OMW decolorization with the degradation of high molecular-mass polyphenols [47, 51]. Furthermore, high correlations were detected for decolorization not only versus laccase activity for majority of the fungi examined $\left(r^{2}=0.83-0.99\right.$, in the cases of G. carnosum, H. croceus, Ph. chrysosporium, and Pleurotus spp.), but also versus $\mathrm{MnP}\left(r^{2}\right.$ = 0.66-0.99, for A. biennis, G. carnosum, $H$. erinaceus, and I. lacteus $)$ and versus MnIP $\left(r^{2}=0.83-0.99\right.$, in the cases of G. carnosum, I. lacteus, and Ph. chrysosporium). In the past, OMW decolorization was associated with laccase production for other white-rot fungi as well [24, 32, 52].

As regards the phytotoxicity evaluation, A. biennis was again the best performer by demonstrating 30\% increase of the germination index with respect to untreated OMW, while G. carnosum, I. lacteus, and $H$. erinaceus provided significantly lower values $(15 \%-18 \%)$. In these cases, increase of plant seed germination was particularly slow during the first 1 to 2 weeks of incubation, which is in line with previous reports on $P$. ostreatus and P. eryngii achieving 19\%-27\% increase of germination index towards the end of the growth period on OMW [32]. On the other hand, the other four strains produced a very low increase in seed germinability $(<10 \%)$ that could be possibly attributed to the formation of phenoxy radicals and/or quinonoids that are more toxic than the initial phenolic compounds $[53,54]$. In general, toxicity of OMW was associated with the presence of aromatic compounds of low molecular weight and with synergistic inhibition caused by phenolics [55-57]. Increase in seed germination was significantly correlated with reduction in phenolics and decolorization $\left(r^{2}=0.84\right.$ and 0.71 , resp., in cumulative calculations made for all strains tested); this high correlation with the former factor is in accordance with past reports on the germination of Triticum and Lepidium plant seeds in OMW treated by L. edodes and Pleurotus spp., respectively $[24,32]$.

When the results presented above were reassessed by taking into consideration the time needed for obtaining the final values for total phenols reduction, decolorization, and increase of plant seed germination, classification of selected strains did not change considerably. For example, A. biennis was again significantly more efficient for all parameters evaluated; that is, it demonstrated a daily average of 3.70 percentage units of total phenols reduction, 2.56 percentage units of decolorization, and 1.20 percentage units of seed germination increase; Pleurotus spp., G. carnosum, H. croceus, and $I$. lacteus followed as regards total phenols reduction (with values of 2.35-2.53), I. lacteus for decolorization (2.24), and G. carnosum, I. lacteus, and $H$. erinaceus for seed germination increase (0.60-0.70).

It is particularly noteworthy that when the experimental data from all eight fungi were assembled and evaluated vis-à-vis their total phenol reduction, decolorization, plant seed germination increase, and enzymes activity (Table 5), then biomass and germination index showed statistically significant correlations in all comparisons $\left(r^{2}=0.55-0.88\right.$ and $r^{2}=0.51-0.88$, resp.). Laccase production was significantly 


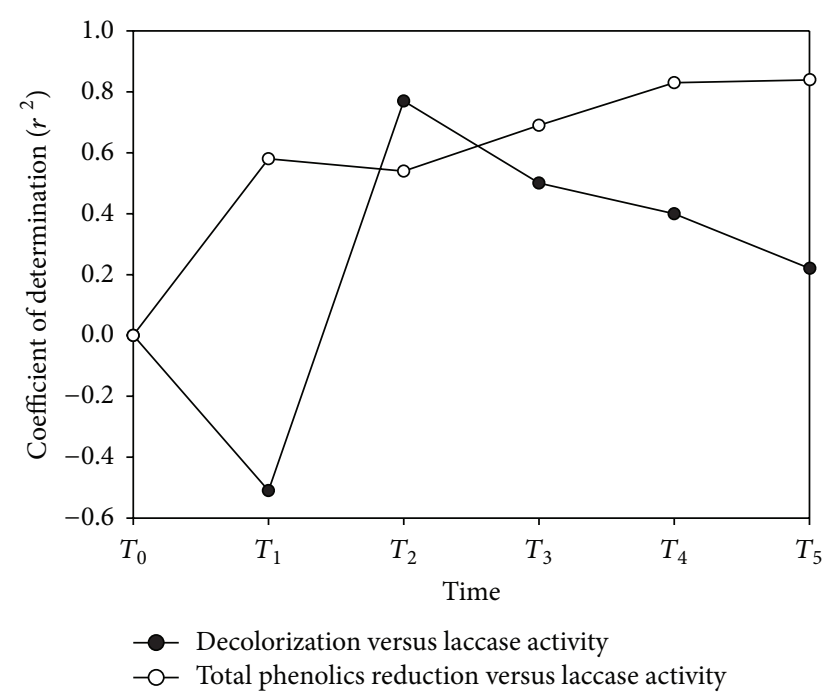

Figure 3: Coefficient of determination $\left(r^{2}\right)$ values for OMW decolorization and total phenolics reduction versus the laccase activities presented by the eight selected macrofungi as calculated from the respective data obtained at five time points $\left(T_{1}\right.$ to $\left.T_{5}\right)$ during their entire growth period.

correlated with total phenolics reduction and decolorization (Figure 3), and so was decolorization with $\operatorname{MnIP}\left(r^{2}=0.46\right)$. Last, MnP, and MnIP activities were also correlated $\left(r^{2}=\right.$ $0.55)$.

\section{Conclusions}

All wood-rot macrofungi selected for the purposes of this study were particularly effective in colonizing and degrading OMW through an efficient enzyme-producing mechanism. Especially A. biennis and secondly I. lacteus, G. carnosum, and Pleurotus spp. achieved total phenol reduction and decolorization values, which were significantly correlated with laccase and/or peroxidases activities. Enhancement of plant seed germination was less pronounced but it demonstrated high correlation with the other two biodegradation parameters. This approach seems to be suitable (alone or in combination with other techniques) in the development of a sustainable methodology leading to the efficient treatment of OMW.

\section{Conflict of Interests}

The authors declare that there is no conflict of interests regarding the publication of this paper.

\section{Acknowledgments}

This research has been cofinanced by European Union (European Social Fund-ESF) and Greek national funds through the Operational Program "Education and Lifelong Learning" of the National Strategic Reference Framework (NSRF)Research Funding Program entitled "Metagenomics of ligninolytic microorganisms-Bioconversion of plant by-products into high-added value products" (THALIS-UOA-MIS 377062). Drs. P. Baldrian and Y. -J. Yao have kindly provided part of the biological material used for the purposes of the study.

\section{References}

[1] M. Hafidi, S. Amir, and J. C. Revel, "Structural characterization of olive mill waster-water after aerobic digestion using elemental analysis, FTIR and C-13 NMR," Process Biochemistry, vol. 40, no. 8, pp. 2615-2622, 2005.

[2] S. Ntougias, F. Gaitis, P. Katsaris, S. Skoulika, N. Iliopoulos, and G. I. Zervakis, "The effects of olives harvest period and production year on olive mill wastewater properties-evaluation of Pleurotus strains as bioindicators of the effluent's toxicity," Chemosphere, vol. 92, no. 4, pp. 399-405, 2013.

[3] G. Ouzounidou, G. I. Zervakis, and F. Gaitis, "Raw and microbiologically detoxified olive mill waste and their impact on plant growth," Terrestrial \& Aquatic Environmental Toxicology, vol. 4, no. 1, pp. 21-38, 2010.

[4] M. I. Badawy, F. El. Gohary, M. Y. Ghaly, and M. E. M. Ali, "Enhancement of olive mill wastewater biodegradation by homogeneous and heterogeneous photocatalytic oxidation," Journal of Hazardous Materials, vol. 169, no. 1-3, pp. 673-679, 2009.

[5] P. Baldrian, G. I. Zervakis, V. Merhautova, S. Ntougias, C. Ehaliotis, and F. Nerud, "The use of hydroxyl-radical-generating systems for the treatment of olive mill wastewaters," Folia Microbiologica, vol. 51, no. 4, pp. 337-341, 2006.

[6] C. Belaid, M. Kallel, M. Khadhraou, G. Lalleve, B. Elleuch, and J. F. Fauvarque, "Electrochemical treatment of olive mill wastewaters: removal of phenolic compounds and decolourization," Journal of Applied Electrochemistry, vol. 36, no. 10, pp. 1175-1182, 2006.

[7] P. Jarboui, B. Hadrich, N. Gharsallah, and E. Ammar, "Olive mill wastewater disposal in evaporation ponds in Sfax (Tunisia): moisture content effect on microbiological and physical chemical parameters," Biodegradation, vol. 20, no. 6, pp. 845-858, 2009.

[8] S. Crognale, F. Federici, and M. Petruccioli, " $\beta$-glucan production by Botryosphaeria rhodina on undiluted olive-mill wastewaters," Biotechnology Letters, vol. 25, no. 23, pp. 20132015, 2003.

[9] A. D’Annibale, G. G. Sermanni, F. Federici, and M. Petruccioli, "Olive-mill wastewaters: a promising substrate for microbial lipase production," Bioresource Technology, vol. 97, no. 15, pp. 1828-1833, 2006.

[10] S. Laconi, G. Molle, A. Cabiddu, and R. Pompei, "Bioremediation of olive oil mill wastewater and production of microbial biomass," Biodegradation, vol. 18, no. 5, pp. 559-566, 2007.

[11] M. J. López, J. Moreno, and A. Ramos-Cormenzana, "Xanthomonas campestris strain selection for xanthan production from olive mill wastewaters," Water Research, vol. 35, no. 7, pp. 1828-1830, 2001.

[12] S. Ntougias, K. Bourtzis, and G. Tsiamis, "The microbiology of olive mill wastes," BioMed Research International, vol. 2013, Article ID 784591, 16 pages, 2013.

[13] E. Kalmıs, N. Azbar, H. Yıldız, and F. Kalyoncu, "Feasibility of using olive mill effluent (OME) as a wetting agent during the cultivation of oyster mushroom, Pleurotus ostreatus, on wheat straw," Bioresource Technology, vol. 99, no. 1, pp. 164-169, 2008. 
[14] S. Papanikolaou, M. Galiotou-Panayotou, S. Fakas, M. Komaitis, and G. Aggelis, "Citric acid production by Yarrowia lipolytica cultivated on olive-mill wastewater-based media," Bioresource Technology, vol. 99, no. 7, pp. 2419-2428, 2008.

[15] C. Pozo, M. V. Martínez-Toledo, B. Rodelas, and J. GonzálezLópez, "Effects of culture conditions on the production of polyhydroxyalkanoates by Azotobacter chroococcum $\mathrm{H} 23$ in media containing a high concentration of alpechin (wastewater from olive oil mills) as primary carbon source," Journal of Biotechnology, vol. 97, no. 2, pp. 125-131, 2002.

[16] S. Bellou, A. Makri, D. Sarris et al., "The olive mill wastewater as substrate for single cell oil production by Zygomycetes," Journal of Biotechnology, vol. 170, pp. 50-59, 2014.

[17] G. Zervakis, P. Yiatras, and C. Balis, "Edible mushrooms from olive mill wastes," International Biodeterioration \& Biodegradation, vol. 38, no. 3-4, pp. 237-243, 1996.

[18] G. I. Zervakis, G. Koutrotsios, and P. Katsaris, "Composted versus raw olive mill waste as substrates for the production of medicinal mushrooms: an assessment of selected cultivation and quality parameters," BioMed Research International, vol. 2013, Article ID 546830, 13 pages, 2013.

[19] P. Baldrian, "Fungal laccases-occurrence and properties," FEMS Microbiology Reviews, vol. 30, no. 2, pp. 215-242, 2006.

[20] K. E. Hammel and D. Cullen, "Role of fungal peroxidases in biological ligninolysis," Current Opinion in Plant Biology, vol. 11, no. 3, pp. 349-355, 2008.

[21] A. T. Martinez, M. Speranza, F. J. Ruiz-Duenas et al., "Biodegradation of lignocellulosics: microbial, chemical, and enzymatic aspects of the fungal attack of lignin," International Microbiology, vol. 8, no. 3, pp. 195-204, 2005.

[22] N. Azbar, A. Bayram, F. Ayes, M. Ayesn, S. Fusun, and A. Ozer, "A review of waste management options in olive oil production," Critical Reviews in Environmental Science and Technology, vol. 34, no. 3, pp. 209-247, 2004.

[23] M. S. Fountoulakis, S. N. Dokianakis, M. E. Kornaros, G. G. Aggelis, and G. Lyberatos, "Removal of phenolics in olive mill wastewaters using the white-rot fungus Pleurotus ostreatus," Water Research, vol. 36, no. 19, pp. 4735-4744, 2002.

[24] A. D’Annibale, M. Ricci, D. Quaratino, F. Federici, and M. Fenice, "Panus tigrinus efficiently removes phenols, color and organic load from olive-mill wastewater," Research in Microbiology, vol. 155, no. 7, pp. 596-603, 2004.

[25] F. E. Ergül, S. Sargin, G. Öngen, and F. V. Sukan, "Dephenolisation of olive mill wastewater using adapted Trametes versicolor," International Biodeterioration and Biodegradation, vol. 63, no. 1, pp. 1-6, 2009.

[26] A. J. F. S. Matos, R. M. F. Bezerra, and A. A. Dias, "Screening of fungal isolates and properties of Ganoderma applanatum intended for olive mill wastewater decolourization and dephenolization," Letters in Applied Microbiology, vol. 45, no. 3, pp. 270-275, 2007.

[27] O. Yesilada, S. Sik, and M. Sam, "Biodegradation of olive oil mill wastewater by Coriolus versicolor and Funalia trogii: effects of agitation, initial COD concentration, inoculum size and immobilization," World Journal of Microbiology and Biotechnology, vol. 14, no. 1, pp. 37-42, 1997.

[28] G. Aggelis, D. Iconomou, M. Christou et al., "Phenolic removal in a model olive oil mill wastewater using Pleurotus ostreatus in bioreactor cultures and biological evaluation of the process," Water Research, vol. 37, no. 16, pp. 3897-3904, 2003.

[29] P. Blánquez, G. Caminal, M. Sarra, M. T. Vicent, and X. Gabarrell, "Olive oil mill waste waters decoloration and detoxification in a bioreactor by the white rot fungus Phanerochaete flavidoalba," Biotechnology Progress, vol. 18, no. 3, pp. 660-662, 2002.

[30] M. Kissi, M. Mountadar, O. Assobhei et al., "Roles of two whiterot basidiomycete fungi in decolorisation and detoxification of olive mill waste water," Applied Microbiology and Biotechnology, vol. 57, no. 1-2, pp. 221-226, 2001.

[31] G. Olivieri, A. Marzocchella, P. Salatino, P. Giardina, G. Cennamo, and G. Sannia, "Olive mill wastewater remediation by means of Pleurotus ostreatus," Biochemical Engineering Journal, vol. 31, no. 3, pp. 180-187, 2006.

[32] S. Ntougias, P. Baldrian, C. Ehaliotis et al., "Biodegradation and detoxification of olive mill wastewater by selected strains of the mushroom genera Ganoderma and Pleurotus," Chemosphere, vol. 88, no. 5, pp. 620-626, 2012.

[33] G. Zervakis, A. Philippoussis, S. Ioannldou, and P. Diamantopoulou, "Mycelium growth kinetics and optimal temperature conditions for the cultivation of edible mushroom species on lignocellulosic substrates," Folia Microbiologica, vol. 46, no. 3, pp. 231-234, 2001.

[34] G. Zervakis and C. Balis, "A pluralistic approach on the study of Pleurotus species, with emphasis on compatibility and physiology of the European morphotaxa," Mycological Research, vol. 100, no. 6, pp. 717-731, 1996.

[35] V. L. Singleton and J. A. Rossi, "Colorimetry of total phenolics with phosphomolybdic phosphotungstic acid reagents," The American Journal of Enology and Viticulture, vol. 16, pp. 144158, 1965.

[36] F. Zucconi, A. Pera, M. Forte, and M. de Bertoldi, "Evaluating toxicity of immature compost," BioCycle, vol. 22, no. 2, pp. 5457, 1981.

[37] R. Bourbonnais and M. G. Paice, "Oxidation of non-phenolic substrates: an expended role for laccase in lignin biodegradation," FEBS Letters, vol. 267, no. 1, pp. 99-102, 1990.

[38] T. T. Ngo and H. M. Lenhoff, "A sensitive and versatile chromogenic assay for peroxidase and peroxidase-coupled reactions," Analytical Biochemistry, vol. 105, no. 2, pp. 389-397, 1980.

[39] A. Tsioulpas, D. Dimou, D. Iconomou, and G. Aggelis, "Phenolic removal in olive oil mill wastewater by strains of Pleurotus spp. in respect to their phenol oxidase (laccase) activity," Bioresource Technology, vol. 84, no. 3, pp. 251-257, 2002.

[40] A. Mekki, A. Dhouib, and S. Sayadi, "Changes in microbial and soil properties following amendment with treated and untreated olive mill wastewater," Microbiological Research, vol. 161, no. 2, pp. 93-101, 2006.

[41] T. K. Kirk and R. L. Farrell, "Enzymatic "combustion": the microbial degradation of lignin," Annual Review of Microbiology, vol. 41, pp. 465-505, 1987.

[42] R. Casa, A. D’Annibale, F. Pieruccetti, S. R. Stazi, G. G. Sermanni, and B. L. Cascio, "Reduction of the phenolic components in olive-mill wastewater by an enzymatic treatment and its impact on durum wheat (Triticum durum Desf.) germinability," Chemosphere, vol. 50, no. 8, pp. 959-966, 2003.

[43] G. Aggelis, C. Ehaliotis, F. Nerud, I. Stoychev, G. Lyberatos, and G. I. Zervakis, "Evaluation of white-rot fungi for detoxification and decolorization of effluents from the green olive debittering process," Applied Microbiology and Biotechnology, vol. 59, no. 23, pp. 353-360, 2002.

[44] C. F. Thurston, "The structure and function of fungal laccases," Microbiology, vol. 140, no. 1, pp. 19-26, 1994. 
[45] U. Tomati, E. Galli, G. Dilena, and R. Buffone, "Induction of laccase in Pleurotus ostreatus mycelium grown in olive oil wastewaters," Agrochimica, vol. 35, pp. 275-279, 1991.

[46] J. Perez, T. de la Rubia, O. Ben Hamman, and J. Martinez, "Phanerochaete flavido-alba laccase induction and modification of manganese peroxidase isoenzyme pattern in decolorized olive oil mill wastewaters," Applied and Environmental Microbiology, vol. 64, no. 7, pp. 2726-2729, 1998.

[47] N. Zouari and R. Ellouz, "Toxic effect of coloured olive compounds on the anaerobic digestion of the olive oil mill effluent in UASB-like reactors," Journal of Chemical Technology and Biotechnology, vol. 66, pp. 414-420, 1996.

[48] A. Jaouani, M. G. Tabka, and M. J. Penninckx, "Lignin modifying enzymes of Coriolopsis polyzona and their role in olive oil mill wastewaters decolourisation," Chemosphere, vol. 62, no. 9, pp. 1421-1430, 2006.

[49] A. D’Annibale, C. Crestini, V. Vinciguerra, and G. G. Sermanni, "The biodegradation of recalcitrant effluents from an olive mill by a white-rot fungus," Journal of Biotechnology, vol. 61, no. 3, pp. 209-218, 1998.

[50] A. Jaouani, S. Sayadi, M. Vanthournhout, and M. J. Penninckx, "Potent fungi for decolourisation of olive oil mill wastewaters," Enzyme and Microbial Technology, vol. 33, no. 6, pp. 802-809, 2003.

[51] S. Sayadi, N. Allouche, M. Jaoua, and F. Aloui, "Detrimental effects of high molecular-mass polyphenols on olive mill wastewater biotreatment," Process Biochemistry, vol. 35, no. 7, pp. 725-735, 2000.

[52] A. A. Dias, R. M. Bezerra, and A. N. Pereira, "Activity and elution profile of laccase during biological decolorization and dephenolization of olive mill wastewater," Bioresource Technology, vol. 92, no. 1, pp. 7-13, 2004.

[53] L. Martirani, P. Giardina, L. Marzullo, and G. Sannia, "Reduction of phenol content and toxicity in olive oil mill waste waters with the ligninolytic fungus Pleurotus ostreatus," Water Research, vol. 30, no. 8, pp. 1914-1918, 1996.

[54] M. Saavedra, E. Benitez, C. Cifuentes, and R. Nogales, "Enzyme activities and chemical changes in wet olive cake after treatment with Pleurotus ostreatus or Eisenia fetida," Biodegradation, vol. 17, no. 1, pp. 93-102, 2006.

[55] R. Capasso, G. Cristinzio, A. Evidente, and F. Scognamiglio, "Isolation, spectroscopy and selective phytotoxic effects of polyphenols from vegetable waste waters," Phytochemistry, vol. 31, no. 12, pp. 4125-4128, 1992.

[56] M. D. Greca, P. Monaco, G. Pinto, A. Pollio, L. Previtera, and F. Temussi, "Phytotoxicity of low-molecular-weight phenols from olive mill waste waters," Bulletin of Environmental Contamination and Toxicology, vol. 67, no. 3, pp. 352-359, 2001.

[57] A. Fiorentino, A. Gentili, M. Isidori et al., "Environmental effects caused by olive mill wastewaters: toxicity comparison of low-molecular-weight phenol components," Journal of Agricultural and Food Chemistry, vol. 51, no. 4, pp. 1005-1009, 2003. 

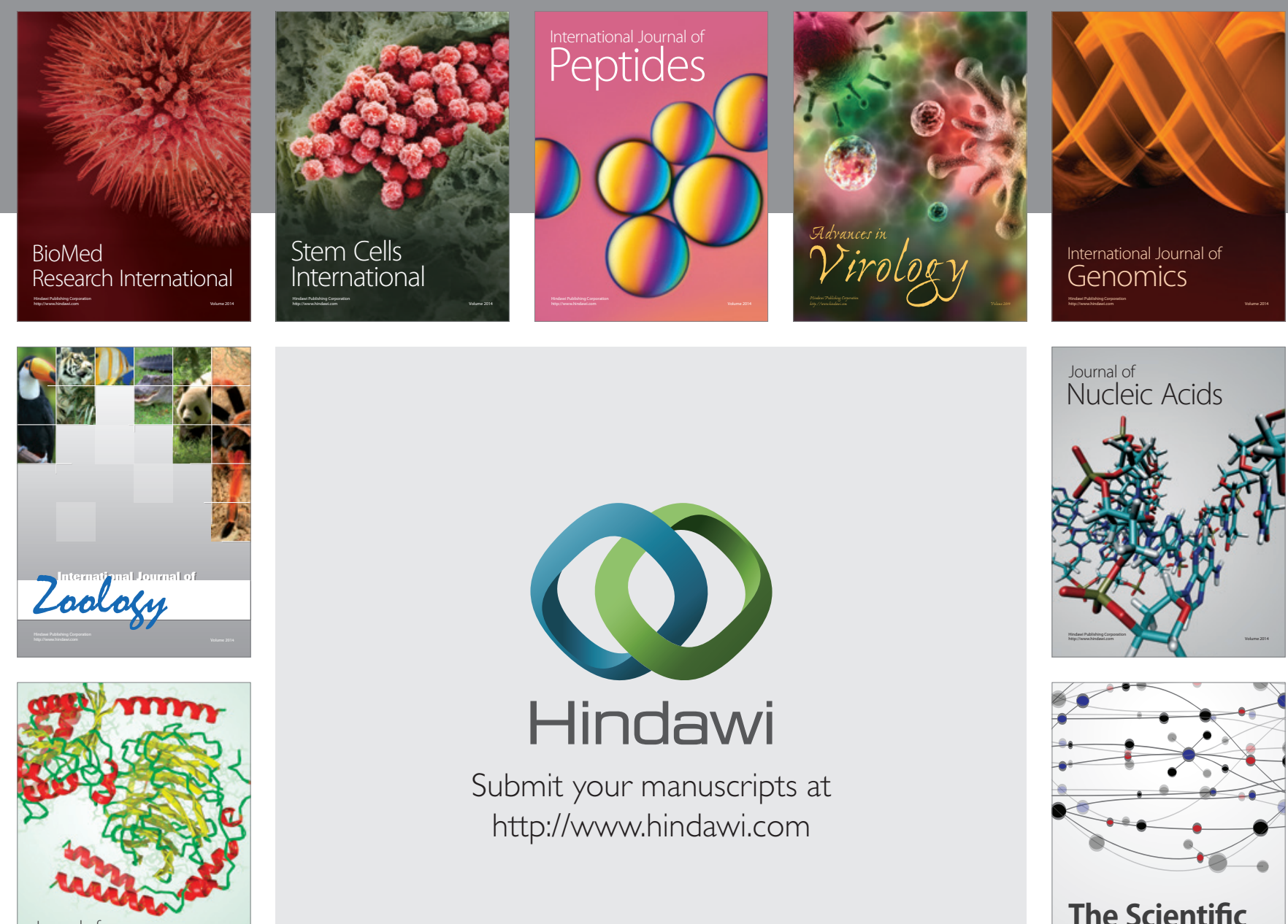

Submit your manuscripts at

http://www.hindawi.com

Journal of
Signal Transduction
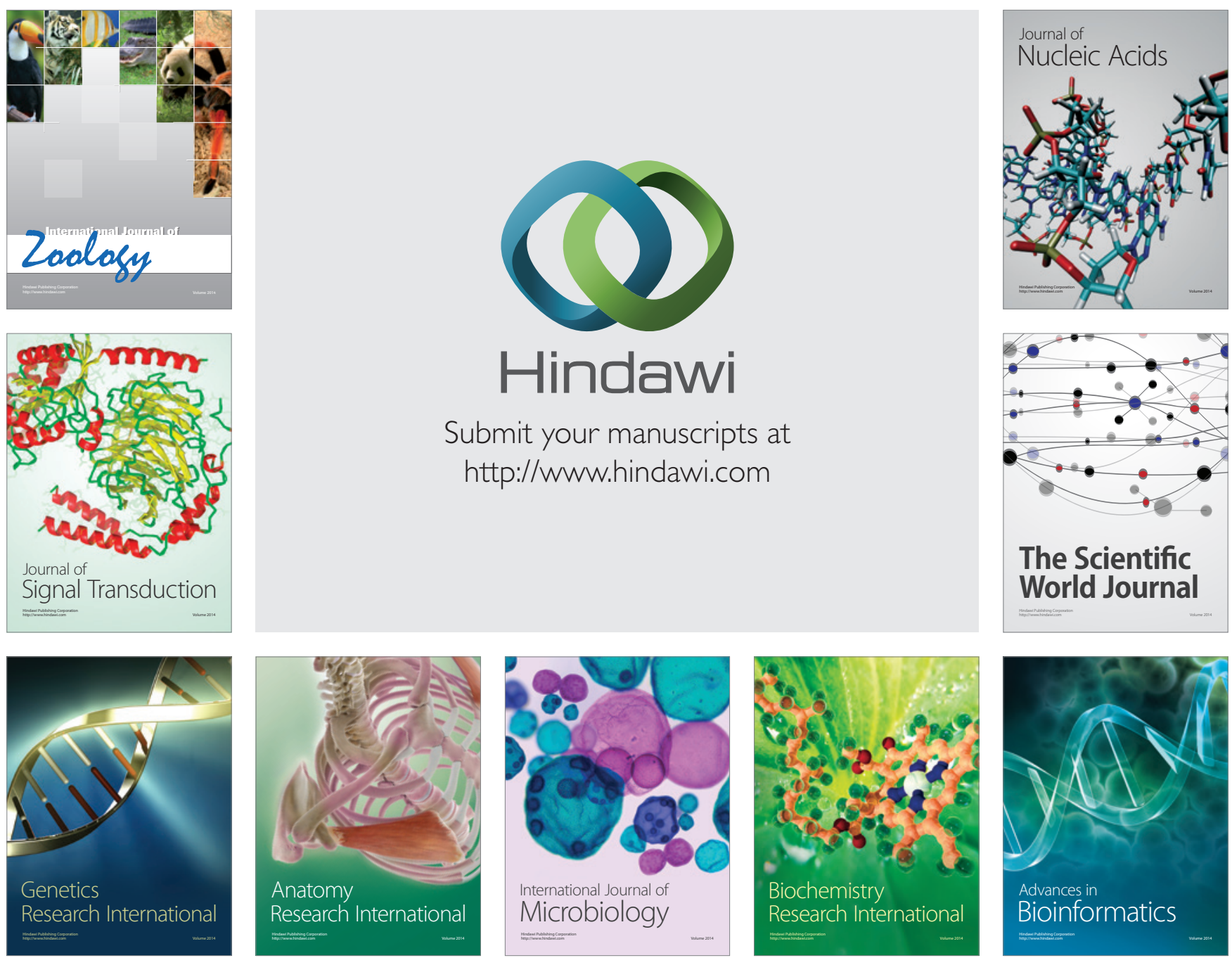

The Scientific World Journal
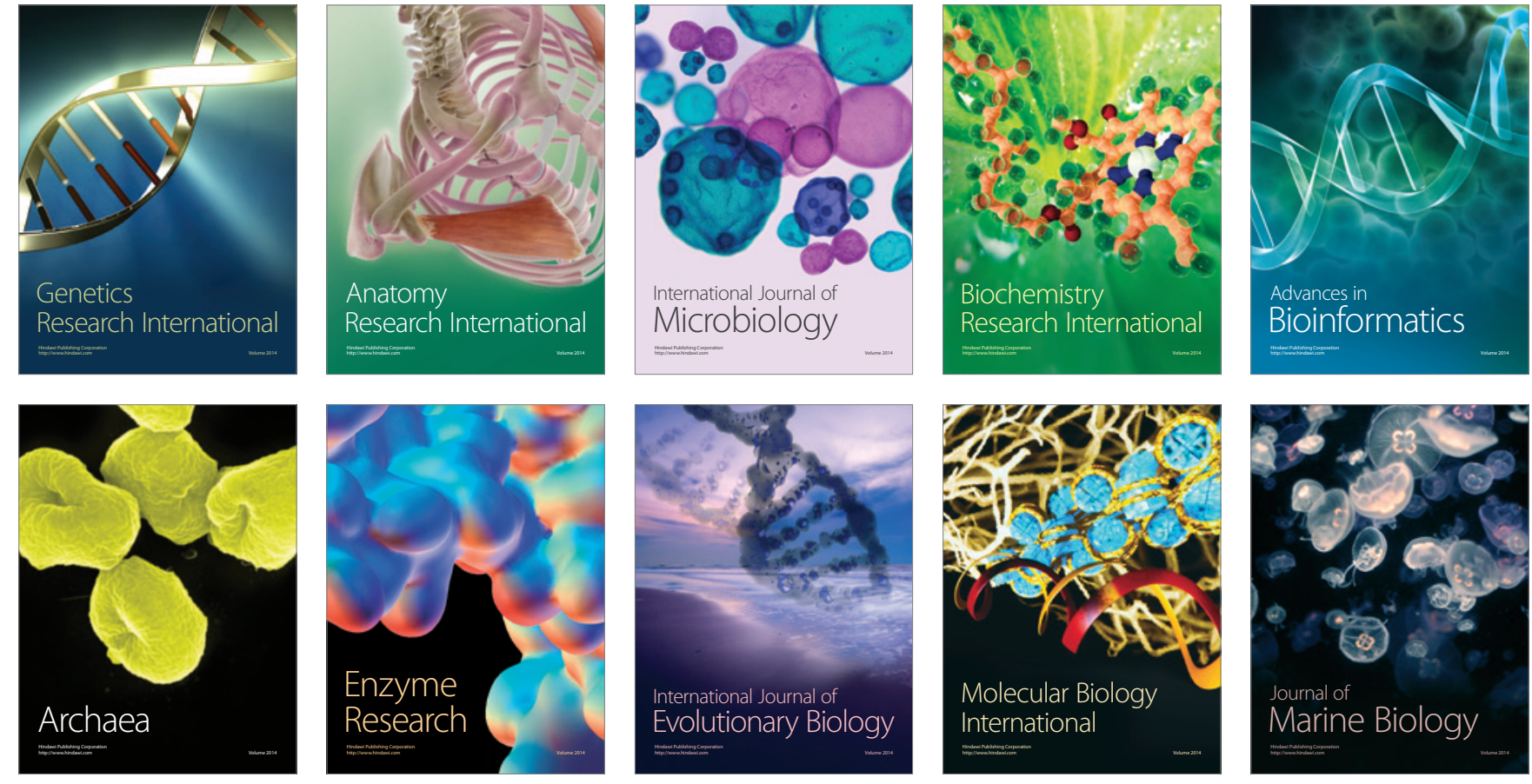